\title{
Charge-Mediated Localization of Conjugated Polythiophenes in Zwitterionic Model Cell
}

\section{Membranes}

Judith E. Houston, ${ }^{\dagger}$ Mario Kraft,, Ian Mooney, ${ }^{\dagger}$ Ann E. Terry,,${ }^{\S}$ Ullrich Scherf ${ }^{\ddagger}$ and Rachel C. $\operatorname{Evans}^{\dagger *}$

School of Chemistry and CRANN, University of Dublin, Trinity College, College Green, Dublin 2, Ireland.

₹Macromolecular Chemistry Group (buwmakro) and Institute for Polymer Technology, Bergische Universität Wuppertal, Gauss-Str. 20, D-42119 Wuppertal, Germany. ISIS, STFC, Rutherford Appleton Laboratory, Didcot, Oxon, OX11 0QX, U.K.

ABSTRACT. The selective engineering of conjugated polyelectrolyte (CPE)-phospholipid interfaces is poised to play a key role in the design of advanced biomedical and biotechnological devices. Herein, we report a strategic study to investigate the relationship between the charge of the CPE side-group and their association with zwitterionic phospholipid bilayers. The interaction of dipalmitoylphosphatidylcholine (DPPC) phospholipid vesicles with a series of poly(thiophene)s bearing zwitterionic, cationic or anionic terminal groups (P3Zwit, P3TMAHT and P3Anionic, respectively) has been probed. Although all CPEs showed an affinity for the 
zwitterionic vesicles, the calculated partition coefficients determined using photoluminescence spectroscopy suggested preferential incorporation within the lipid bilayer in the order P3Zwit > P3Anionic » P3TMAHT. The polarity probe, Prodan, was used to further qualify the position of the CPE inside the vesicle bilayers via Förster resonance energy transfer (FRET) studies. The varying proximity of the CPEs to Prodan was reflected in the Stern-Volmer quenching constants and decreased in the order P3Anionic > P3TMAHT » P3Zwit. Dynamic light scattering measurements showed an increase in hydrodynamic diameter of the DPPC vesicles upon addition of each poly(thiophene), but to the greatest extent for P3Anionic. Small-angle neutron scattering studies also revealed that P3Anionic specifically increased the thickness of the headgroup region of the phospholipid bilayer. Epi-fluorescence and atomic force microscopy imaging showed that P3TMAHT formed amorphous agglomerates on the vesicle surface, P3Zwit was buried throughout the bilayer and that P3Anionic formed a shell of protruding chains around the surface, which promoted vesicle fusion. The global data indicate three distinctive modes of interaction for the poly(thiophene)s within DPPC vesicles, whereby the nature of the association is ultimately controlled by the pendant charge-group on each CPE chain. Our results suggest that charge-mediated self-assembly may provide a simple and effective route to design luminescent CPE probes capable of specific localization within phospholipid membranes.

KEYWORDS: conjugated polyelectrolytes, polythiophenes, membrane-polyelectrolyte interactions, membrane probes, phospholipids, surfactants 


\section{INTRODUCTION}

Within every biological system the cell membrane facilitates the transportation of materials, energy and information between the interior and exterior cell environment, achieved by modification of their structure or phase. Although the precise phase composition of cell membranes remains a topic of wide debate, it is believed to consist of mobile liquid-ordered lipid domains (or rafts) embedded within a fluid phase. ${ }^{2.3}$ Homogenous phospholipid large unilamellar vesicles (LUVs) are commonly used as models for complex plasma membranes ${ }^{4}$ and have recently shown potential as sophisticated drug delivery vehicles ${ }^{5}$, and as nanoreactors. ${ }^{6}$ Despite their considerable differences to live cell membranes, the interaction of probe molecules with LUVs can provide vital information on a range of variables such as binding affinity, orientation or localization and the local environment. ${ }^{4}$ Fluorescent membrane probes (e.g. organic dyes, ${ }^{7.8}$ quantum dots, , fluorescently-labeled phospholipids ${ }^{10}$ and proteins") are particularly attractive for this purpose, providing a means of directly monitoring membrane probe interactions with both spatial and temporal resolution..$^{12,13}$

The ideal fluorescent probe combines high chemical and photochemical stability, precise localization within the membrane bilayer, without becoming internalized within the cell, as well as environment-responsive optical properties that provide information about their molecular surroundings (e.g. through spectral shifts, fluorescence intensity or lifetime changes, etc.). ${ }^{14}$ However, physical parameters, such as hydration, fluidity, and dipole and transmembrane potentials, change dramatically across the membrane bilayer; ${ }^{15}$ as such knowledge and control of where a probe specifically locates is also crucial. Typical strategies to regulate the localization of probes within cell membranes involve complex synthetic design, requiring covalent-bonding of molecular dyes to biomolecules" or the use of large phospholipid analogues..$^{10}$ is $\mathrm{A}$ more facile 
approach exploits interfacial interactions between the probe and the phospholipid bilayer, governed by intermolecular forces such as van der Waals, electrostatic co-assembly and hydrophobic interactions. ${ }^{15}$ The challenge for this strategy, however, is to design probes which, through a delicate balance of intermolecular forces, are able to localize specifically at a targeted location within a phospholipid bilayer.

Conjugated polyelectrolytes (CPEs) are attractive membrane probes due to their watersolubility and environment-16, ${ }^{17}$ and aggregation-responsive fluorescence. ${ }^{18}$ Moreover, the introduction of ionic terminal groups onto their lateral chains can be exploited to additionally impart the charge-mediated behavior characteristic of polyelectrolytes into their rich properties..$^{19}$ ${ }^{20}$ Studies of the interaction of CPEs with phospholipid vesicles have delivered a broad range of potential applications not only for cell biology, for example cell imaging, ${ }^{21}$ fabrication of sensing devices $^{17}$ and formation of biocidal agents, ${ }^{22}$ but also for biotechnological applications, including the optimization of power generation in microbial fuel cells..$^{23}$ The extent of CPE incorporation within phospholipid vesicles has previously been achieved by varying the nature and number of charges on the phospholipid head-group,,$^{24}$ as well as the nature and number of side-group charges $^{24}$ and length of the polymer chain..$^{25,26}$ Cosa et al. investigated the effect of varying the chain length of anionic poly(phenylene ethynylene)s (PPEs) and their interaction with phospholipids of different head-group charge..$^{25}$ Regardless of the PPE chain length, the anionic CPE showed no interaction with negatively-charged phospholipid vesicles and associated only to a small extent with zwitterionic phospholipids. In contrast, strong interactions were observed with the cationic phospholipid, 1,2-dioleoyl-3-trimethylammonium-propane (DOTAP), with vesicle fusion being reported exclusively for the longest chain-length PPE. A comparative study between cationic PPEs and the anionic phospholipid, 1,2-dipalmitoyl-sn-glycero-3- 
phosphoglycerol (DPPG), commonly used as model membranes for bacterial cells, found that the extent of membrane perturbation of the CPEs decreased with increasing CPE size. ${ }^{26}$ Mammalian cell membranes predominantly consist of zwitterionic phospholipids, ${ }^{27}$ which possess both positive and negative charges in their polar head-groups. However, to date, limited studies exist regarding the effect of zwitterionic surfactants, generally phospholipids, on the solution behavior of CPEs. ${ }^{24}{ }^{25}$ Recently, it was shown that the nature of the surfactant (cationic, anionic, zwitterionic, non-ionic) when added to a solution of the zwitterionic CPE, poly[3-(N-(4sulfonato-1-butyl)- $N, N$-diethylammonium)hexyl-2,5-thiophene], had a significant effect on the optical and structural properties of the zwitterion..$^{28}$ Specific ionic associations between charged groups and non-specific interactions, such as structural rearrangements to accommodate incorporation of the surfactant tails, were proposed to govern the nanoscale organization of the CPE. Furthermore, this zwitterionic poly(thiophene) derivative offers a unique advantage over the anionic and cationic alternatives due to the absence of mobile counterions ${ }^{16}$ which are known to cause fluorescent quenching, an undesirable side effect in the design of fluorescent membrane probes.

To the best of our knowledge, no study to date has probed the interaction between zwitterionic phospholipids and CPEs containing the same conjugated backbone, but different charges on the pendant side-groups. To address this, here we investigate the co-assembly of zwitterionic dipalmitoylphosphatidylcholine (DPPC) LUVs with a series of poly(thiophene)s bearing zwitterionic, cationic or anionic terminal groups (P3Zwit, P3TMAHT and P3Anionic, respectively), Figure 1. Recently, we have shown that P3Anionic is uniquely capable of sequentially detecting three phase transitions in zwitterionic model cell membranes via distinctive changes to its emission properties..$^{29}$ There was significant evidence to suggest that the 
charge of the anionic poly(thiophene) mediates its incorporation within the motionally-restrictive zwitterionic phospholipid head-group region, where the sensitivity to membrane order is believed to be the greatest. This preliminary study revealed the potential opportunity to mediate the interaction of a CPE with a phospholipid bilayer via judicious selection of the pendant charge-group on the CPE. Here, using a combination of optical, microscopy and small-angle scattering (light, neutrons) measurements, we will demonstrate that the localization of the probe within different zones of the lipid bilayer can be controlled simply by changing the nature of the ionic charge on the side chains of the CPE. Our findings provide insight into the potential of charge-mediation to control the membrane perturbation of CPEs and may prove valuable in the design of more effective membrane probes, as well as in the development of improved theranostic platforms.
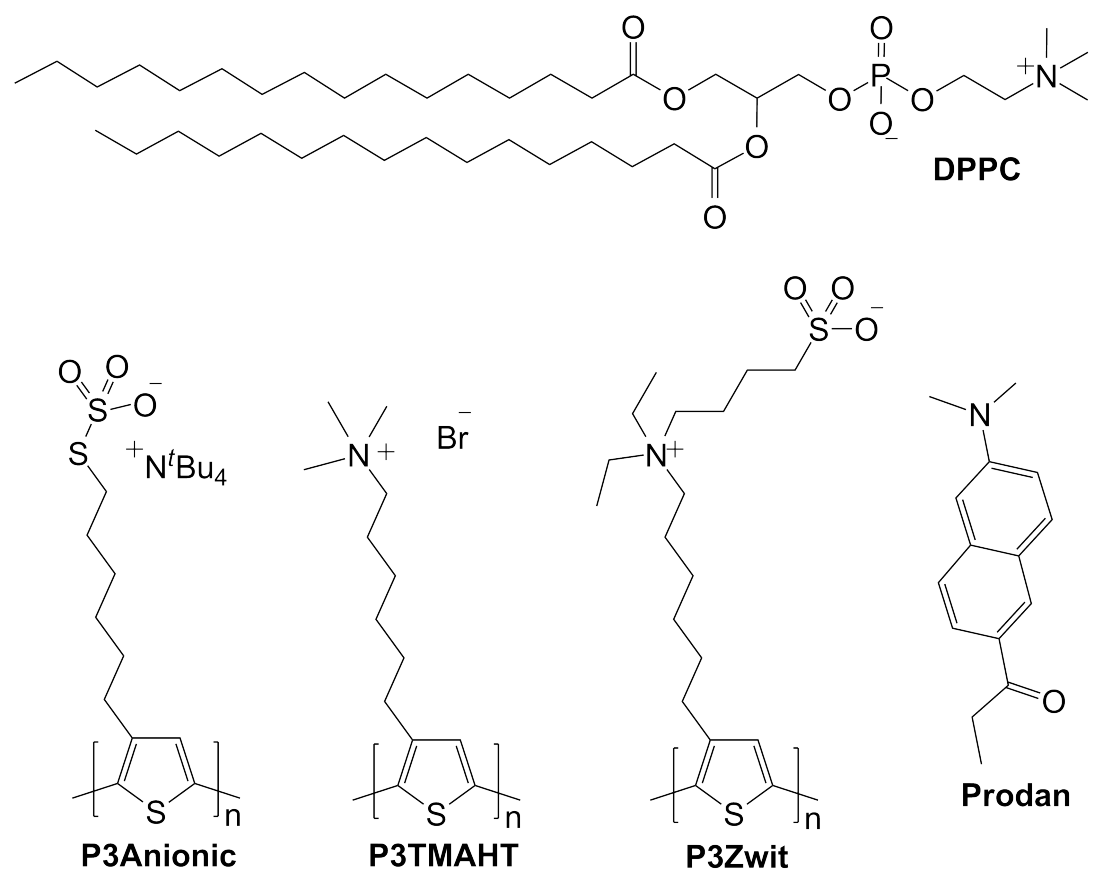

Figure 1. Chemical structures of the phospholipid, DPPC, the three poly(thiophene)s investigated, P3Anionic, P3Zwit and P3TMAHT, and the polarity probe, Prodan. 


\section{EXPERIMENTAL SECTION}

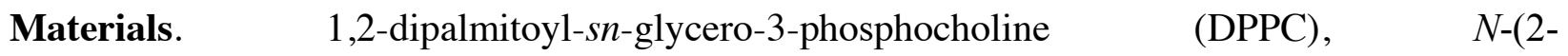
hydroxyethyl)piperazine- $N$ '-(2-ethanesulfonic acid) HEPES ( $\geq 99.5 \%)$ and 6-propionyl-2dimethylaminonaphthalene (Prodan) $(\geq 98.0 \%)$ were purchased from Sigma Aldrich. Sodium chloride and chloroform were purchased from Fisher Scientific. Deuterium oxide ( $>99.9 \%)$ was purchased from Apollo Scientific Limited. All materials were used as received. Poly[3-(6trimethylammoniumhexyl)thiophene] (P3TMAHT) ${ }^{30} \quad$ poly[3-(6-sulfothioatehexyl)thiophene)] (P3Anionic) $)^{31}$ and poly[3-(N-(4-sulfonato-1-butyl)- $N, N$-diethylammonium)hexyl-2,5-thiophene) (P3Zwit, also known as P3SBDEAHT) ${ }^{28}$ were synthesized as previously reported ${ }^{2 x, 80,31}$ and have number-averaged molecular weights, $M_{n}$, of 12,000, 5,000 and 12,000 g mol-1, respectively, with PDIs of 1.1, as measured for the parent bromohexyl precursors. Unless otherwise stated, all buffers utilized had the same ionic strength: $10 \mathrm{mM}$ HEPES, $30 \mathrm{mM} \mathrm{NaCl}$, pH 7.3.

Vesicle Preparation. DPPC powder was dissolved in chloroform to obtain a final concentration of $40 \mathrm{mg} \mathrm{mL}$. The solvent was then evaporated while rotating the sample vial to create a thin film on the vial wall. Films were placed under vacuum for at least $1 \mathrm{~h}$ to remove excess solvent. Phospholipid films were hydrated to a final concentration of $20 \mathrm{mM}$ with either buffer or buffer solution containing dissolved Prodan $(4 \mu \mathrm{M})$. The phospholipid suspensions were then subjected to 15 cycles of freeze-thaw-sonicate, where freeze indicates $10 \mathrm{~min}$ in the freezer, thaw indicates $1 \mathrm{~min}$ in a water bath at $40-50{ }^{\circ} \mathrm{C}$, and sonicate indicates $5 \mathrm{~min}$ of sonication at $40-50{ }^{\circ} \mathrm{C}$. The phospholipid mixtures were subsequently extruded 15 times through a polycarbonate membrane (100 $\mathrm{nm}$ pore size). During the extrusion process the samples were maintained above the gelliquid transition temperature of DPPC $\left.\left(42^{\circ} \mathrm{C}\right)\right)^{32}$ The vesicle suspensions were stored at $4{ }^{\circ} \mathrm{C}$ before use, if they were not used immediately. 
Titration Procedure. For each measurement, stock solutions of the CPE were mixed with stock solutions of the DPPC LUVs to obtain the required charge ratio between the CPE monomer units and the zwitterionic DPPC molecules. The molecular weights, $M_{\mathrm{w}}$, of each CPE monomer unit are 302.3, 519.9 and $373.6 \mathrm{~g} \mathrm{~mol}{ }^{-1}$ for P3TMAHT, P3Anionic and P3Zwit, respectively.

Absorption and Emission Spectroscopy. UV/Vis absorption and fluorescence spectra were recorded at room temperature on a Shimadzu UV2401 PC UV/Vis scanning spectrometer and a Fluorolog-3 (Horiba Jobin Yvon) spectrophotometer, respectively. Emission and excitation spectra were corrected for the wavelength response of the system using correction factors supplied by the manufacturer. Samples were measured in $1 \mathrm{~cm}$ pathlength quartz cuvettes.

Dynamic Light Scattering (DLS) and Zeta Potential $(\xi)$. DLS and $\zeta$ measurements were performed using a Zetasizer Nano series nano-ZS (Malvern Instruments, U.K.). For DLS the instrument was set in the back-scattering configuration $\left(175^{\circ}\right)$. Cumulant analysis of the correlation function was used to determine the mean Z-average hydrodynamic diameter $\left(D_{\mathrm{n}}\right)$ and the polydispersity (PDI) of each sample. ${ }^{33}$ For the $\zeta$ measurements electrophoretic mobilities were recorded by a Laser-Doppler facility in the DLS unit. The $\zeta$ was calculated from the electrophoretic mobility, $\mu$, using the Henry correction to Smoluchowski's equation: ${ }^{34}$

$$
\zeta=\mu\left(\frac{4 \pi \eta}{\varepsilon^{0}}\right)
$$

where $\eta$ is the solvent viscosity and $\varepsilon^{0}$ is its static dielectric permittivity. Equation 1 places no restrictions on the particle shape, except for the assumption that the radius of curvature of the surface is much greater than the Debye screening length, $x^{-1}$. Smoluchowski's approximation holds because the electrical double layer thickness surrounding the vesicles, $\delta$, is much lower than $D_{\mathfrak{}} / 2 .{ }^{35}$ 
Small-Angle Neutron Scattering (SANS). SANS measurements were carried out on the LOQ beamline at the ISIS Pulsed Neutron Source (STFC Rutherford Appleton Laboratory, Didcot, U.K.). An incident wavelength range of 2.2-10 ̊ separated by time-of-flight with a sample-todetector distance of $4.1 \mathrm{~m}$ yielded a simultaneous $q$-range of $\sim 0.009-0.24 \AA^{-1}$, as $q=$ $(4 \pi / \lambda) \sin (\theta / 2)$, where $\lambda$ is the wavelength and $\theta$ the scattering angle. Samples were prepared in deuterium oxide $\left(\mathrm{D}_{2} \mathrm{O}\right)$ to provide good neutron scattering contrast. Each raw scattering data set was corrected for the detector efficiency, sample transmission and background scattering and converted to scattering cross-section data $(\partial \Sigma / \partial \Omega$ vs $q)$ using the instrument-specific software Mantid ${ }_{{ }^{36}}$ These data were placed on an absolute scale $\left(\mathrm{cm}^{-1}\right)$ through reference to the scattering from a standard sample (a solid blend of hydrogenated and perdeuterated polystyrene) in accordance with established procedures ${ }^{37}$ The SANS scattering functions $I(q)$ were initially interpreted using scaling concepts. ${ }^{38}$ The scattering intensity may be considered to scale as:

$$
\frac{1}{c} I(q) \approx q^{-\alpha}
$$

where $c$ is the concentration and the exponent $\alpha$ may be interpreted in terms of an arbitrary particle shape: $\alpha=1$ for separated rods; $\alpha=2$ for sheet-like particles; $\alpha=4$ for smooth 3 dimensional particles, for which the scattering would also level off at low $q$. The low- $q$ data arise from the structure of the vesicles themselves, while the high- $q$ data derive from the internal structure of the phospholipid bilayer, ${ }^{39}$ and can be used to probe changes that take place in response to the CPEs. The thickness of the vesicle bilayer was first analyzed using the modified Kratky-Porod (MKP) approach, ${ }^{, 0}$ which relates the radius of gyration to the thickness of the bilayer and is independent of other model fitting.

The scattering functions were then fit using non-linear least-squares analysis to a Core-MultiShell model using the SasView programme..$^{41,42}$ In this model, four shells were used to represent 
the phospholipid head-group and hydrophobic chain regions of the inner leaflet, and the phospholipid head-group and hydrophobic chain regions of the outer leaflet. The core of the model contains the same solution background as exists outside the vesicles. Neutron scattering lengths and volumes of the various components in the system that were used to calculate the scattering length densities (SLDs) in the model are provided in Table S1 in the Supporting Information. The scattering intensity from the four-shell model is given by: ${ }^{42}$

$$
I(q)=A\left[\sum_{i=1}^{4} \frac{3 V_{i}\left(\rho_{i}-\rho_{i}\right) j_{1}\left(q d_{i}\right)}{q d_{i}}\right]^{2} S(q)+b k g
$$

where $A$ is a scaling factor, $b k g$ is a constant background, $\varrho_{i}$ are the SLDs of each shell, $\varrho_{0}$ is the neutron SLD of the core, which is equal to that of the buffer solution, $V_{\mathrm{i}}$ is the volume of each shell and $j_{1}(x)$ is the Bessel function. The $d_{\mathrm{i}}(i=1,2,3,4)$ are the radii of the shells in the model, being the radius, the thickness of the head-group and the chain region of the inner leaflet, and the thickness of the head-group and the chain region of the outer leaflet, respectively. $S(q)$ is the structure factor for intervesicle interactions and is equal to unity $(S(q)=1)$ for the dilute vesicles solutions measured in this experiment. The fits were checked to have absolute SANS intensities consistent with the known sample concentrations (1 vol\% dry material).

Atomic Force Microscopy (AFM). AFM measurements were performed using an Asylum Research MFP-3D ${ }^{\mathrm{TM}}$ instrument mounted on an anti-vibration plinth, in the tapping mode at room temperature under ambient conditions. Higher resolution AFM measurements were performed using sharp diamond tips, which were a kind gift from Adama Innovations. All raw AFM images were visualized and analyzed using the Gwyddion 2.31 software.

Determination of Partition Coefficients. The partition coefficient, $K_{\mathrm{p}}$, provides information about the partitioning of the CPEs between the phospholipid and water phases of the phospholipid vesicles, and can be determined from the fluorescence intensity, depending on 
whether quenching or enhancement is observed. For fluorescent molecules that display an increase in emission intensity, $I$, upon the addition of phospholipid vesicles, the partition coefficient, $K_{\mathrm{p}}$, is determined from: $:^{43.4}$

$$
I=\frac{I_{w}+K_{p} \gamma_{L}[L] I_{\max }}{1+K_{p} \gamma_{L}[L]}
$$

where $\gamma_{\llcorner}$is the molar volume of phospholipid, $I_{w}$ is the emission intensity of the CPE in the absence of the vesicles, and $I_{\max }$ is the maximum emission intensity that can be achieved upon addition of vesicles to the CPE. A plot of emission intensity at a fixed wavelength against phospholipid concentration, $[L]$, will be hyperbolic if $I_{m a x}>I_{w}$. In this case the initial slope at $[L]=$ $0 \mathrm{~mol} \mathrm{dm}{ }^{3}$ is given by:

$$
\text { Slope }=K_{p} \gamma_{L}\left(I_{\max }-I_{w}\right)
$$

Alternatively, if a fluorescent molecule is quenched upon titration with phospholipid vesicles, $K_{\mathrm{p}}$ can be calculated from: ${ }^{: 5}$

$$
\frac{I_{\max }}{I}=1+K_{p} \alpha
$$

where $\alpha=V_{\mathrm{L}} / V_{\mathrm{w}}, V_{\mathrm{L}}$ is the volume of phospholipid and $V_{\mathrm{w}}$ is the total volume. $V_{\mathrm{L}}$ can be calculated by taking the volume of a molecule of DPPC as $1148 \AA^{3{ }^{36}}$ Eq. 4 can be equated to Eq. 6 if the limit of emission intensity, $I_{m a x}$, is assumed to be zero. ${ }^{44}$ A plot of $I_{\text {max }} / I v s \alpha$ should be linear, where the slope is equal to the partition coefficient, $K_{p}$.

Förster Resonance Energy Transfer. The efficiency of energy transfer by dipolar donoracceptor $(D-A)$ interactions described by Förster theory is given by:t

$$
E=\frac{R_{0}^{6}}{R_{0}^{6}+r^{6}}
$$

where $r$ is the distance between the donor and the acceptor and $R_{0}$ is the Förster distance at which the efficiency of energy transfer, $E$, is $50 \% . R_{0}$ is given by: 


$$
R_{0}^{6}=\frac{9000(\ln 10) \kappa^{2} \phi_{D}}{128 \pi^{5} N_{A} n^{4}} J_{D A}
$$

where $\mathcal{X}^{2}$ is the orientation factor of two dipoles interacting and is assumed to be 0.472 for the gel state of DPPC vesicles. ${ }^{48} \Phi_{\mathrm{D}}$ is the fluorescence quantum yield of the donor in absence of the acceptor, $\Phi_{\mathrm{D}}$ is 0.23 for Prodan in DPPC vesicles at $25^{\circ} \mathrm{C},{ }^{48} n$ is the average refractive index of the host phospholipid bilayer and $N_{\mathrm{A}}$ is Avogadro's number. $J_{\mathrm{DA}}(\lambda)$ is the spectral overlap integral between the donor emission and acceptor absorption spectra.

$$
J_{D A}(\lambda)=\int_{0}^{\infty} F_{D}(\lambda) \varepsilon_{A}(\lambda) \lambda^{4} d \lambda
$$

where $F_{\mathrm{D}}(\lambda)$ is the fluorescence spectrum of the donor normalized so that $\int_{0}^{\infty} F_{D}(\lambda) \mathrm{d} \lambda=1$ and $\varepsilon_{\mathrm{A}}(\lambda)$ is the molar absorption coefficient of the acceptor.

\section{RESULTS AND DISCUSSION}

Photophysical Characterization. Due to their inherent charges, electrostatic association between the poly(thiophene)s and the zwitterionic phospholipid head-groups is expected. The UV/Vis absorption and fluorescence spectra of P3TMAHT, P3Zwit and P3Anionic were studied upon titration with increasing concentrations of DPPC vesicles in HEPES buffer solution (30 $\mathrm{mM} \mathrm{NaCl}, \mathrm{pH} 7.3$ ) at $25{ }^{\circ} \mathrm{C}$. For solutions of the pure $\mathrm{CPE}$, single absorption bands were observed, centered at $\lambda_{\text {abs }}=431 \mathrm{~nm}$ for P3Zwit and P3TMAHT and $435 \mathrm{~nm}$ for P3Anionic. Upon titration with DPPC vesicles, a blue-shift and an increase in the absorbance was observed for all CPEs (Figure 2 and Figure S1a). For P3Anionic red-edge band broadening was also observed which is most likely attributed to an increase in conjugation length of the poly(thiophene);49 however scattering effects arising from the addition of nanometre sized vesicles to the solution cannot be excluded. 

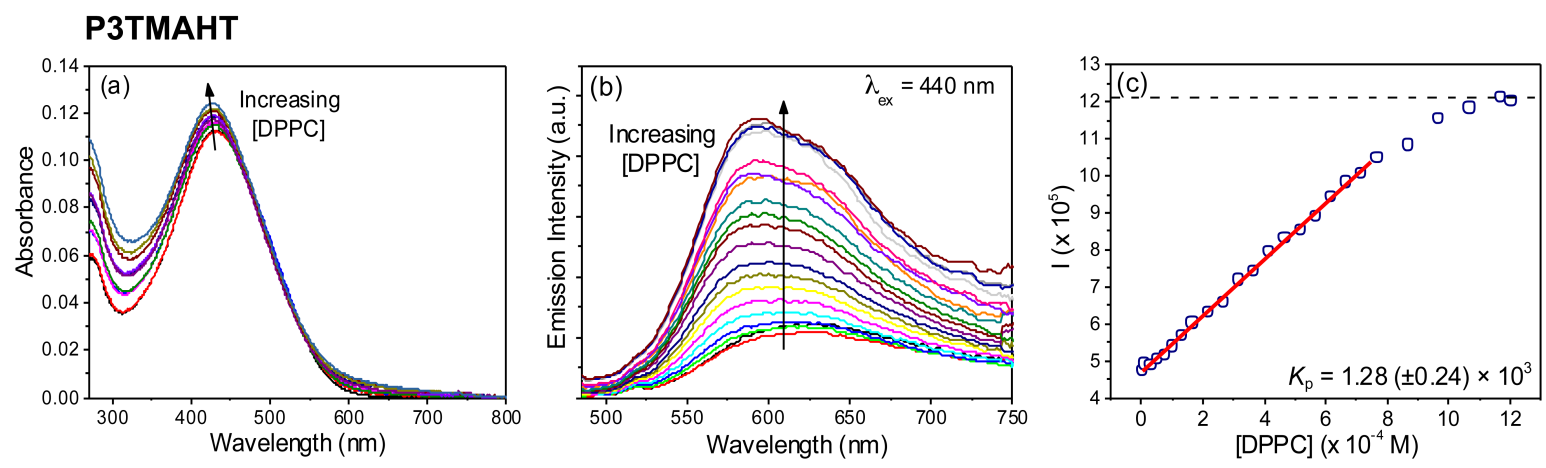

P3Zwit
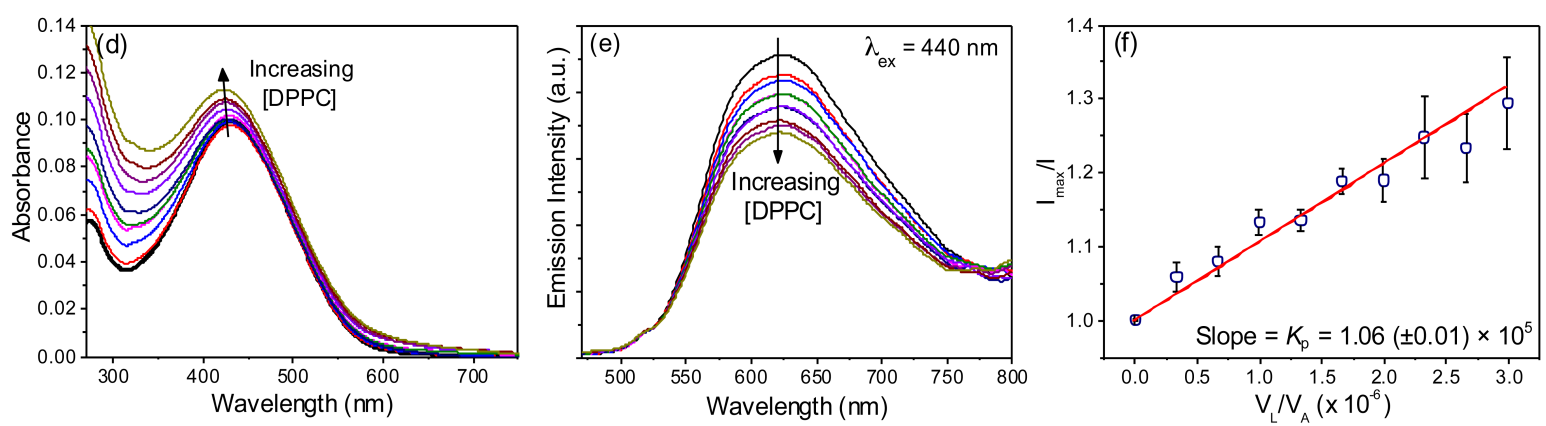

Figure 2. (a, d) UV/Vis absorption and (b, e) emission spectra of P3TMAHT $\left(3.96 \times 10^{-5} \mathrm{M}\right.$ (r.u.)) and P3Zwit $\left(1.93 \times 10^{-5} \mathrm{M}\right.$ (r.u.)), respectively, upon titration with DPPC vesicles (0 - 9.60 $\times 10^{-5} \mathrm{M}$ and $0-4.08 \times 10^{-5} \mathrm{M}$ for P3TMAHT and P3Zwit, respectively). (c) Plot of emission intensity, $I$, at $600 \mathrm{~nm} v s$ DPPC concentration for determination of $K_{\mathrm{p}}$ for P3TMAHT. (f) Plot of $I_{\max } / I$ vs phospholipid volume fraction for determination of $K_{\mathrm{p}}$ for P3Zwit.

Each CPE also exhibits a single broad emission band with $\lambda_{\mathrm{em}}$ centered at 625, 607 and $625 \mathrm{~nm}$ for P3TMAHT, P3Anionic and P3Zwit, respectively. The addition of DPPC vesicles into a buffered solution of P3TMAHT results in the appearance of vibronic structure in the emission spectrum, a significant blue-shift $\left(\Delta_{\mathrm{em}}=625-595 \mathrm{~nm}\right)$ and an increase in emission intensity, reaching a three-fold enhancement by a 3:1 DPPC:CPE charge ratio (Figure 2b). In contrast, P3Zwit and P3Anionic exhibit a quenching of the emission intensity, with a smaller blue-shift in 
$\lambda_{\mathrm{cm}}$ from 625 to $622 \mathrm{~nm}$ for P3Zwit (Figure 2e), and 607 to $604 \mathrm{~nm}$ for P3Anionic (see Figure S1b). The excitation spectra are in excellent agreement with the absorption spectra (Figure S2). The measured $\lambda_{\mathrm{asc}}$ and $\lambda_{\mathrm{em}}$ for the CPEs in the absence and presence of DPPC vesicles (at 1:1 charge ratio of CPE:DPPC), and the calculated $K_{\mathrm{r}}$, are summarized in Table 1.

Table 1. UV/Vis absorption $\left(\lambda_{\mathrm{man}}\right)$ and emission $\left(\lambda_{\mathrm{cm}}\right)$ maxima for CPEs before and after the addition of DPPC vesicles (1:1 charge ratio) in HEPES buffer solution (30 mM NaCl, $\mathrm{pH} 7.3)$, the calculated partition coefficients, $K_{\mathrm{p}}$ and the calculated number of repeat units (and chains) of CPE expected to associate with each DPPC vesicle.

\begin{tabular}{|c|c|c|c|c|c|c|}
\hline \multicolumn{3}{|c|}{$\lambda_{\text {stos }}(\mathrm{nm})$} & \multicolumn{2}{|c|}{$\lambda_{\mathrm{em}}(\mathrm{nm})$} & \multirow[b]{2}{*}{$K_{\mathrm{p}}$} & \\
\hline CPE & $\begin{array}{l}\text { without } \\
\text { DPPC }\end{array}$ & $\begin{array}{l}\text { with } \\
\text { DPPC }\end{array}$ & $\begin{array}{l}\text { without } \\
\text { DPPC }\end{array}$ & $\begin{array}{l}\text { with } \\
\text { DPPC }\end{array}$ & & $\begin{array}{l}\text { Number of CPE } \\
\text { r.u. (chains) per } \\
\text { DPPC vesicle }\end{array}$ \\
\hline P3Anionic & 431 & 426 & 607 & 604 & $4.86( \pm 0.06) \times 10$ & $8,800(419)$ \\
\hline P3Zwitterionic & 435 & 424 & 625 & 622 & $1.06( \pm 0.01) \times 10^{5}$ & $8,800(180)$ \\
\hline P3TMAHT & 435 & 427 & 625 & 595 & $1.28( \pm 0.24) \times 10$ & $8,790(179)$ \\
\hline
\end{tabular}

- The number of CPE r.u. per DPPC vesicle are calculated for a concentration of CPE $=1 \times 10^{\text {s }}$ (r.u.) $\mathrm{M}$ and DPPC $=$ $1 \times 10^{+} \mathrm{M}$ (See SI for calculation details).

Changes in the UV/Vis absorption and emission spectra can be used to probe the mode of interaction between the CPEs and the DPPC vesicles. ${ }^{50}$ In addition, the partition coefficient, $K_{\mathrm{p}}$, provides information about the distribution of the CPE between the phospholipid and water phases of the solutions of phospholipid vesicles, including the approximate number of CPE chains per DPPC vesicle. These values can be determined from the fluorescence intensity depending on whether there is quenching or enhancement, as described in the Experimental section. ${ }^{345}$ The increased emission intensity and blue-shift observed for P3TMAHT is common 
for CPEs when combined with phospholipids,,$^{18,24}$ oppositely-charged,,$^{19,51}$ or neutral surfactants..$^{51,52}$ Such changes have been attributed to the surfactant-induced break-up of the CPE clusters to form new surfactant-CPE aggregates, , $2^{2}$ where the conformation of the CPE changes from an aggregated to more extended state..$^{53}$ The observed emission intensity enhancement and blue-shift in $\lambda_{\max }$ are indicative of higher interchain disorder due to distortion of neighboring thienylene building blocks, which leads to a corresponding decreased conjugative interaction in the deaggregated state..$^{33,54}$

The observed quenching of the P3Zwit and P3Anionic emission upon addition of DPPC vesicles may be the result of the CPEs embedding within the membrane and forming groundstate complexes with the charged phospholipid head-groups. ${ }^{16}$ The large $K_{\mathrm{p}}$ for P3Zwit (1.06 $\left.( \pm 0.01) \times 10^{5}\right)$ suggests that this CPE is buried deep within the bilayer. In contrast, the lower $K_{\mathrm{p}}$ for P3Anionic $\left(4.86( \pm 0.06) \times 10^{4}\right)$ may infer that it is restricted from penetrating as deeply. It is interesting to note that the shorter chain-length poly(thiophene), P3Anionic, $(\sim 8 \mathrm{~nm})$ would typically be expected to penetrate further within the phospholipid bilayer than the longer chainlength, P3Zwit $(\sim 20 \mathrm{~nm}){ }_{.}^{25,26}$ In comparison, P3TMAHT presents the lowest $K_{\mathrm{p}}$ value $(1.28$ $\left.( \pm 0.24) \times 10^{3}\right)$, suggesting that is least effectively incorporated within the phospholipid bilayer. This is the first indication that the charge of the CPE side-chain may play a crucial role in controlling the penetration depth within the zwitterionic bilayers. We note that while the $K_{\mathrm{p}}$ values vary by several orders of magnitude, this does not translate into a significant difference in the estimated average number of repeat conjugated units incorporated per vesicle for each $\mathrm{CPE}$ system (see Table 1). However, this does correspond to a higher density of individual chains per vesicle for P3Anionic due to its lower molecular weight. 
Förster Resonance Energy Transfer (FRET) Studies. Environment-sensitive hydrophobic probes are often used to obtain information about the structure and interactions within biomembranes.15 The emission spectrum of the probe, Prodan, is highly sensitive to the polarity of the local environment, ${ }^{48}$ and was selected to examine more precisely the location of the CPEs within the phospholipid bilayer by FRET. Prodan can be incorporated within the DPPC bilayers via simple titration before or after vesicle preparation, with a calculated partition coefficient of $1.8 \times 10^{s}$ (Figure S3). In water, Prodan exhibits a broad emission band centered at $525 \mathrm{~nm}$, which shifts to $445 \mathrm{~nm}$ upon incorporation/binding with DPPC (Figure 3a) as the origin of the emission shifts from a charge-transfer to a locally-excited state. ${ }^{\sharp 8}$ Visual inspection of the emission spectrum of Prodan in DPPC vesicles (donor) and the absorption spectra of the CPEs (acceptor) reveals excellent overlap, and results in large values for the calculated spectral overlap integral, $J_{\mathrm{DA}}$, as shown in Table 2, which suggest that FRET should take place.

As shown in Figure 3b for P3Zwit (see Figure S4 for P3Anionic and P3TMAHT), following selective excitation of Prodan $\left(\lambda_{\mathrm{ec}}=340 \mathrm{~nm}\right)$, the emission band at $\lambda_{\mathrm{cm}}=435 \mathrm{~nm}$ becomes increasingly quenched upon addition of each CPE, in the preferential order: P3Anionic > P3TMAHT » P3Zwit. Moreover, a congruent increase in the emission intensity at $610 \mathrm{~nm}$, attributed to the poly(thiophene) emission, is observed. The corresponding excitation spectra detected in the CPE emission band reveal a contribution from both the CPE $(\sim 450 \mathrm{~nm})$ and the Prodan excitation $(\sim 360 \mathrm{~nm})$, confirming that Prodan to CPE energy transfer occurs (see Figure 3c for P3Zwit, Figure S5 for P3Anionic and P3TMAHT). The corresponding Stern-Volmer plots for quenching of the Prodan emission by the CPE were shown to be linear (Figure 3d) and the calculated Stern-Volmer constants are collected in Table 2. 

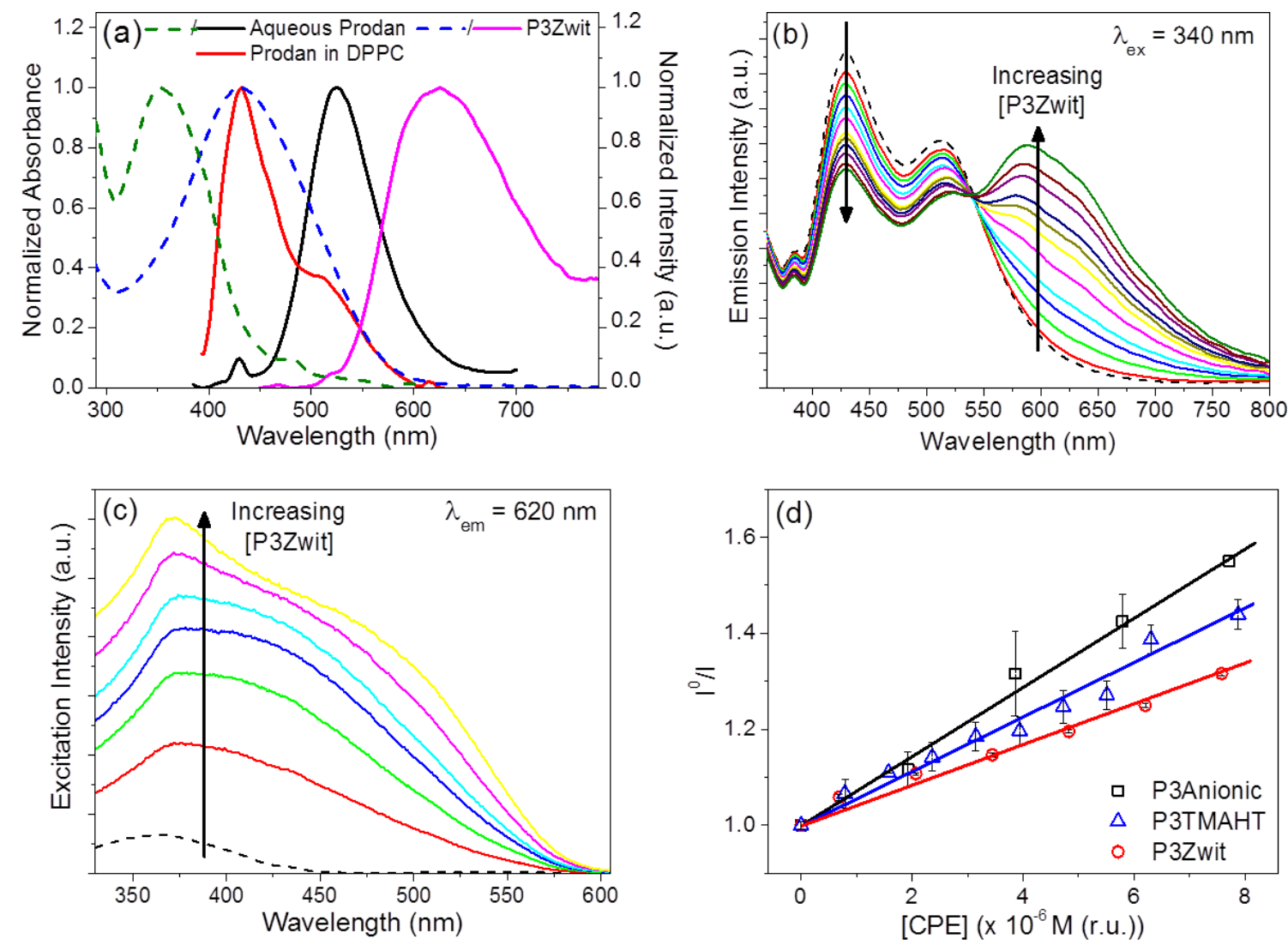

Figure 3. (a) Normalized absorption (dashed lines) and emission (solid lines) spectra of Prodan and P3Zwit in HEPES buffer before and after the addition of DPPC vesicles, $(30 \mathrm{mM} \mathrm{NaCl}, \mathrm{pH}$ 7.3). (b) Emission and (c) excitation spectra of Prodan $\left(2 \times 10^{-8} \mathrm{M}\right)$ in DPPC $\left(1 \times 10^{-4} \mathrm{M}\right)$ vesicles upon the addition of P3Zwit (0-1.45 $\times 10^{-5} \mathrm{M}$ (r.u.)). (d) Stern-Volmer plots of the quenching of Prodan by P3Anionic (black squares), P3TMAHT (blue triangles) and P3Zwit (red circles). To enable direct comparison between CPEs, titrations were performed with a constant concentration of DPPC/Prodan stock solution.

Fluorescence quenching is a powerful technique to obtain topographical information about proteins, nucleic acids and biological membrane systems..$^{16,55}$ However, in systems involving membranes it can be difficult to make quantitative conclusions about the precise $D$ - $A$ distances 
as the donor and/or the quencher are likely to be partitioned between the aqueous phase and the phospholipid phase. However, from the calculated $K_{\mathrm{p}}$ values, we estimate that there will be a $10^{\mathrm{s}}$ and $10^{3}-10^{s}$ fold excess of Prodan and the CPEs, respectively, partitioned within the vesicle membrane relative to the aqueous buffer solution. Moreover, control experiments on the analogous Prodan/CPE systems in the absence of DPPC vesicles reveal that no significant quenching of the Prodan fluorescence at these quencher concentrations (Figure S6 and S7), i.e. energy transfer between Prodan and the CPEs in free buffer solution should make a negligible contribution to the trends presented here. This allows us to make qualitative deductions about the relative position of the acceptor CPEs in relation to Prodan, which is known to preferentially locate at the boundary between the glycerol head-group and the lipid chains of phospholipids.56

The Förster equation ${ }^{\text {s }}$ for resonance energy transfer has its limitations for linear conjugated systems as the point-dipole model cannot adequately account for the spatial distribution of excitations at short donors-acceptor distances. ${ }^{5 s, s 9}$ However, Förster theory can provide a useful tool to compare differences in the energy transfer efficiencies for each system. As the calculated Förster distances, $R_{0}$, are relatively similar for all the CPEs (see Table 2), and FRET efficiencies are highly dependent on the center to center $D$ - $A$ distance, ${ }^{, 7}$ any differences in the $K_{\mathrm{sv}}$ values can be interpreted as the direct result of a change in distance between the Prodan donor and the CPE acceptor. The magnitude of the Stern-Volmer constants indicate that quenching of the Prodan emission by all three CPEs proceeds through a static quenching mode. ${ }^{\infty}$ Interestingly, P3Zwit has the highest $K_{\mathrm{v}}$ and yet the lowest $K_{\mathrm{sv}}\left(3.80( \pm 0.64) \times 10^{4} \mathrm{M}\right.$ (r.u.)-1), suggesting that while this CPE is best incorporated within the DPPC bilayer, it is located at the greatest distance from the glycerol/lipid backbone (where the Prodan is located), potentially deep within the hydrophobic lipid tails. In comparison, the significantly lower $K_{\mathrm{sv}}$ for P3TMAHT and 
P3Anionic $\left(5.55( \pm 1.64) \times 10^{4}\right.$ and $6.38( \pm 2.22) \times 10^{4} \mathrm{M}(\text { r.u. })^{-1}$, respectively $)$ suggest that these poly(thiophene)s are in close proximity to Prodan and thus the glycerol/lipid backbone.

Table 2. Measured molar absorption coefficients for the CPEs, $\varepsilon_{\wedge}$, and calculated spectral overlap integrals, $J_{\mathrm{DA}}$, Förster distances, $R_{0}$, and Stern-Volmer constants, $K_{\mathrm{sv}}$.

\begin{tabular}{|c|c|c|c|c|c|}
\hline Donor & Acceptor & $\begin{array}{l}\varepsilon_{\wedge} \text { at } 430 \mathrm{~nm} \\
\left.(\mathrm{M} \text { (r.u. })^{-\prime} \mathrm{cm}^{-1}\right)\end{array}$ & $\begin{array}{c}J_{D \Lambda}\left(\times 10^{-4}\right. \\
\left.\mathrm{M}(\text { r.u. })^{-!} \mathrm{cm}^{3}\right)\end{array}$ & $R_{0}(\mathrm{~nm})$ & $K_{\mathrm{sv}}\left(\times 10^{4} \mathrm{M}(\text { r.u. })^{-1}\right)$ \\
\hline Prodan & Р3ТМАНТ & 8,483 & 2.46 & 2.82 & $5.55( \pm 1.64)$ \\
\hline Prodan & P3Zwit & 13,193 & 3.84 & 3.04 & $3.80( \pm 0.64)$ \\
\hline Prodan & P3Anionic & 9,242 & 2.85 & 2.89 & $6.38( \pm 2.22)$ \\
\hline
\end{tabular}

Particle Size and Surface Charge Studies. DLS has previously been used to confirm that the integrity of phospholipid vesicles is retained upon the addition of CPEs. ${ }^{241}$ The $Z$-average hydrodynamic diameter $\left(D_{\mathrm{n}}\right)$ of the phospholipid vesicles was measured before and after addition of the CPEs (summarized in Table S3). Pure DPPC vesicles have a narrow size distribution with a mean $D_{\mathrm{n}}$ of $115.3( \pm 2.0) \mathrm{nm}$ and a polydispersity (PDI) of $0.12( \pm 0.01)$. This size closely corresponds to the $100 \mathrm{~nm}$ diameter pore size of the polycarbonate filter used to prepare the vesicles. The vesicle size was unchanged when stored at $4{ }^{\circ} \mathrm{C}$ for 7 days. The pure $\mathrm{CPE}$ samples formed much larger aggregates in solution, with a mean $D_{\text {n }}$ of $393.9( \pm 9.4), 382.8( \pm 36.6)$ and $244.7( \pm 2.5) \mathrm{nm}$ for P3Zwit, P3TMAHT and P3Anionic, respectively, and large size distributions, with PDIs of 0.35 or greater.

Upon titration of the CPEs into a buffered solution of DPPC vesicles, a modest increase in $D_{\mathrm{n}}$ was observed, to $113.5( \pm 0.6), 119.3( \pm 1.5)$ and $130.0( \pm 4.5) \mathrm{nm}$ for P3Zwit, P3TMAHT and P3Anionic, respectively. However, the size distributions remained narrow, with PDIs of 0.11 
$( \pm 0.01), 0.15( \pm 0.01)$ and $0.16( \pm 0.02)$ for P3Zwit, P3TMAHT and P3Anionic, respectively. The DLS size measurements indicate that the DPPC vesicles break-up large CPE aggregates and that the vesicle structures are retained instead of being disrupted into smaller fragments..$^{18}$ It is well accepted that when CPEs (or other molecules) adsorb or penetrate vesicle surfaces there will be a moderate change in $D_{\mathrm{n}}$ of the particle. .4.2.6.6264 $^{2.62}$

Zeta potential $(\zeta)$ measurements were used to determine the effective charge of the vesicle surface, which will change depending on whether electrostatic associations between the CPE and DPPC occur at the vesicle surface or within the phospholipid bilayer. ${ }^{\circ 5}$ The trends in $\zeta$ for DPPC vesicles, pure CPEs and CPE/DPPC mixtures at 1:1 charge ratio prepared in HEPES buffer, $\mathrm{pH} 7.3$, are shown in Figure 4. The $\zeta$ of DPPC vesicles was $-3.3( \pm 0.1) \mathrm{mV}$, close to the literature value of $\sim-4.4 \mathrm{mV}{ }^{66} \mathrm{~A}$ comparable $\zeta$ for P3Zwit was recorded at $-5.6( \pm 0.4) \mathrm{mV}$. As the surface charges of both materials are similar, it is unsurprising that upon combination a similar $\zeta$ of $-7.9( \pm 3.7) \mathrm{mV}$ was observed. In contrast, the cationic CPE, P3TMAHT, had a positive $\zeta$ of $+12.5( \pm 2.8) \mathrm{mV}$. Upon addition of P3TMAHT to DPPC vesicles the $\zeta$ increased dramatically to $+18.1( \pm 1.7) \mathrm{mV}$. Previously, the cationic CPE, poly(allyl $-N, N$-dimethyl $-N$ hexylammonium chloride) was absorbed onto zwitterionic phospholipid vesicles and the positively-charged ammonium ions of the CPE were shown to be exposed to the bulk solution, increasing the surface potential of the vesicles. ${ }^{67}$ These results thus suggest that P3TMAHT covers the outer surface of the zwitterionic vesicle. Pure P3Anionic has a negative $\zeta$ of -28.1 $( \pm 5.2) \mathrm{mV}$, whilst the $\zeta$ of the P3Anionic/DPPC mixture lies between this value and that of DPPC at $-25.4( \pm 1.0) \mathrm{mV}$. Since the DPPC vesicles only partially adopt the $\zeta$ of P3Anionic, it is reasonable to suggest that the $\mathrm{CPE}$ may be moderately penetrating the phospholipid bilayer with chains also protruding from the outer surface. ${ }^{67}$ 


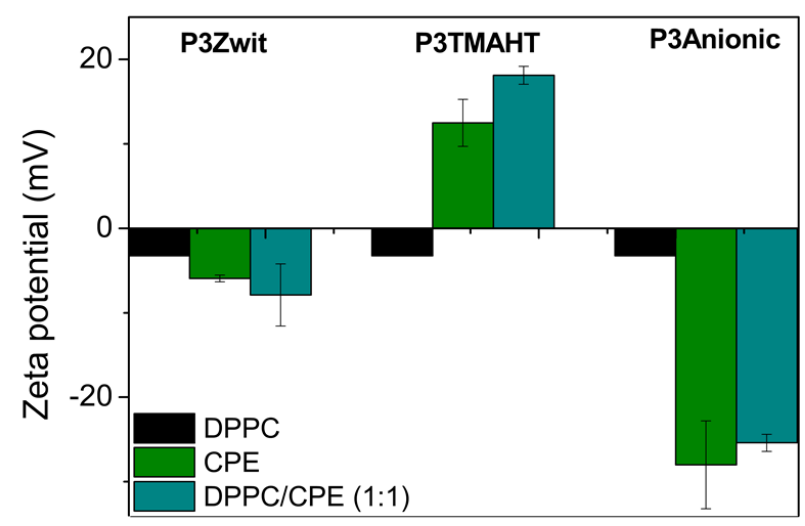

Figure 4. Zeta potentials of DPPC vesicles, CPEs and 1:1 CPE:DPPC charge ratio mixtures (conc. $=4.0 \times 10^{-} \mathrm{M}$ (r.u.)) in HEPES buffer, $30 \mathrm{mM} \mathrm{NaCl}(\mathrm{pH} 7.3)$. Each value is the average of at least three measurements.

Small-Angle Neutron Scattering (SANS). SANS experiments were performed on the $\mathrm{CPE} / \mathrm{DPPC}$ vesicles (in $30 \mathrm{mM} \mathrm{NaCl}$ deuterated water solutions) to obtain deeper insight into the internal nanoscale organization of the vesicle bilayers. The data were initially interpreted using scaling concepts to provide information about the CPE/DPPC aggregate shape (Figure 5a) ${ }^{38}$ The observation window of the SANS experiments ranged from 3.0 to $69.8 \mathrm{~nm}$, which is less than the diameter of isolated vesicles $(\sim 100 \mathrm{~nm})$. However, it does cover the isolated chain length of the fully extended poly(thiophene)s $(7.8-19.6 \mathrm{~nm}){ }^{38}$ This means that fully dissolved rod-like polymers would be expected to exhibit a monatomic - 1 decay, with a Guinier plateau in the low$q$ regime. This was not observed for any of the scattering curves, implying that the pure CPE solutions and the CPE/DPPC samples all contained CPE associations (Figure S10). The low- $q$ data $(q<0.07)$ arise from the structure of the vesicles themselves, while the high- $q$ data $(0.07<q)$ originate from the internal structure of the bilayer ${ }^{39}$ and can be used to probe changes in the structural organization that result from interaction with the CPEs. The low- $q$ regions for DPPC vesicles, P3TMAHT/DPPC and P3Zwit/DPPC mixtures decayed as $\sim q^{2}$, which is typical of 
scattering from a flat rather than spherical bilayer. ${ }^{68}$ This is to be expected considering that the radius of the vesicles is significantly greater than the thickness of the vesicle bilayer. In contrast, the P3Anionic/DPPC samples decayed as $q^{-1.6}$ in the low- $q$ region, shifting to $q^{4.1}$ in the high- $q$ region. This excess scatter at $q=0.05 \AA^{-1}$ could arise from a structure factor resulting from interparticle repulsion between similarly charged poly(thiophene) chains on the vesicle surfaces. ${ }^{69}$. ${ }^{70}$ Interestingly, the high- $q$ "Porod" region $\left(q>0.08 \AA^{-1}\right)$ of the P3Anionic/DPPC and P3Zwit/DPPC scattering profiles decays as $\sim q^{-4}$, consistent with scattering from smooth surfaces. ${ }^{71}$ In contrast, the same region of the P3TMAHT/DPPC profile decays as $\sim q^{-3}$, indicating slightly rougher vesicle surfaces.
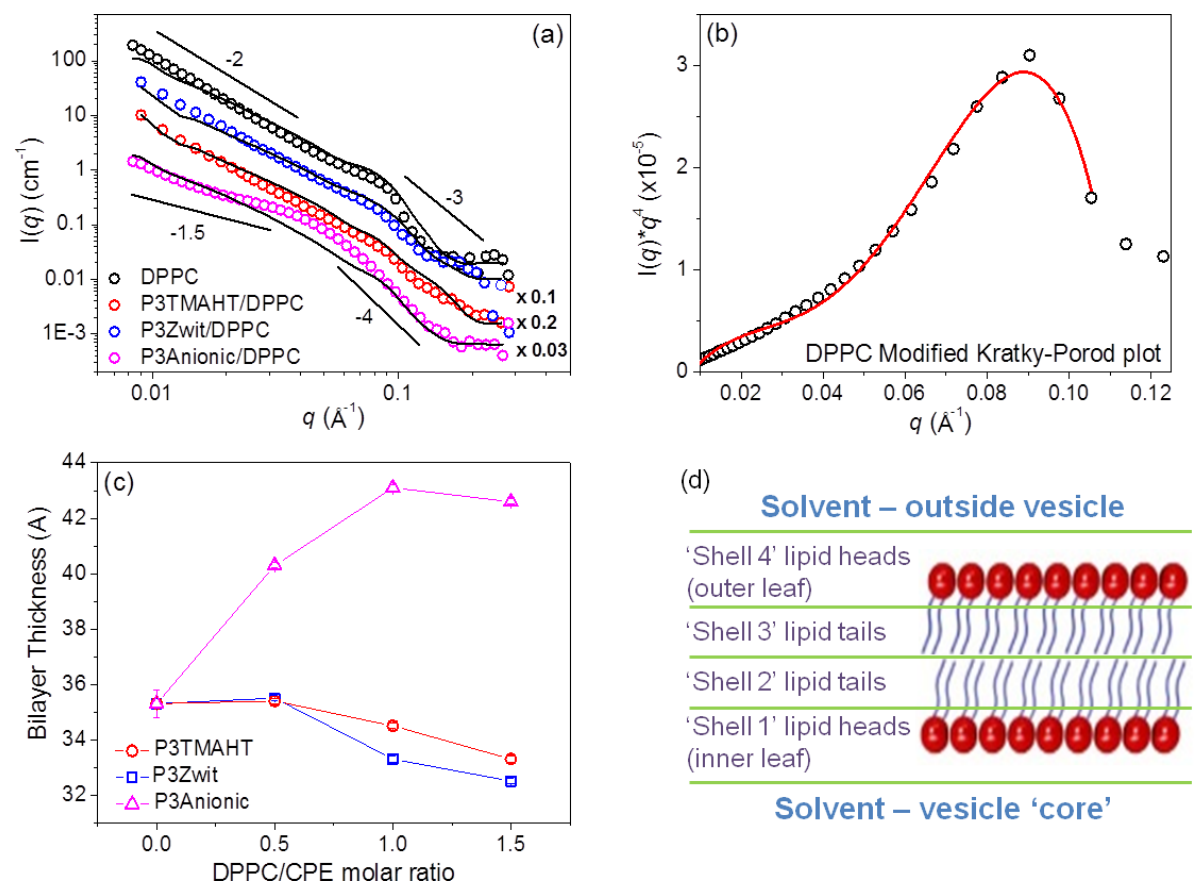

Figure 5. SANS data for DPPC vesicles and mixtures of P3TMAHT/DPPC, P3Zwit/DPPC and P3Anionic/DPPC (1:1 charge ratio, $\left.10 \mathrm{mg} \mathrm{mL}^{-1}\right)$ in $\mathrm{D}_{2} \mathrm{O}$, which have been offset for clarity. The solid lines show the fit to the data using a Core-Multi-Shell model as described in the text. (b) Modified Kratky-Porod (MKP) fit for DPPC vesicles. (c) MKP bilayer thicknesses of DPPC with 
increasing charge ratios of the CPEs. (d) Schematic of the phospholipid bilayer, defining the regions into which the Core-Multi-Shell model is divided.

The thickness of the bilayer was subsequently analyzed using the modified Kratky-Porod (MKP) approach, ${ }^{40}$ which relates the radius of gyration to the thickness of the bilayer and is independent of other model fitting. Pencer and Hallet found that the position of the first local maximum, $q_{\max }$, in the $I(q)^{*} q^{4}$ vs $q$ plot, could be used to obtain the bilayer thickness in unilamellar vesicles, if a homogeneous distribution of coherent neutron scattering length densities (SLDs) within the bilayer is assumed..$^{2}$ The MKP fit for pure DPPC (Figure 5b) gave a calculated bilayer thickness of $35.3( \pm 0.5) \AA$ and the calculated thicknesses for all CPE/DPPC samples at different charge ratios are shown in Figure 5c. (MKP fits for the CPE/DPPC samples at different charge ratios are shown in Figure S11.) The bilayer thicknesses for P3Zwit/DPPC and P3TMAHT/DPPC samples were found to decrease slightly to $33.3( \pm 0.1)$ and $34.5( \pm 0.1) \AA$, respectively, upon reaching a 1:1 charge ratio of CPE:DPPC. In contrast, the P3Anionic/DPPC samples increased in thickness to $43.3( \pm 0.1) \AA$ at the same charge ratio.

The SANS data $\left(0.009-0.27 \AA^{-1}\right)$ were also fit to a Core-Multi-Shell model using a nonlinear least-squares method, including polydispersity and $q$-resolution smearing. ${ }^{42}$ The best fits for the DPPC vesicles and CPE/DPPC samples are shown in Figure 5a and the SLDs used for fitting are summarized in Table S1. In this model, four shells were used to represent the phospholipid head-groups and the hydrophobic chains in the inner and outer regions of the bilayer (Figure 5d). The core of the vesicle is expected to contain the same solvent background as that outside of the vesicle. Due to the negligible binding constants of $\mathrm{Na}^{+}$and $\mathrm{Cl}^{-}$ions $(0.28$ 
and $0.25 \mathrm{M}^{-1}$, respectively) ${ }^{73}$ to zwitterionic vesicles, and the low concentration used (30 mM), ion associations are assumed to be unimportant and $\mathrm{NaCl}$ has not been included in the fits. ${ }^{74}$

The total thickness of the blank DPPC vesicle bilayer with and without CPE using the Core-Multi-Shell model followed the same trend as the MKP fits (Table 3). The DPPC bilayer became slightly thinner upon addition of P3TMAHT from 38.0 to $35.1 \AA$. However, the headgroup regions were thicker in comparison to pure DPPC vesicles. The SLD of the DPPC headgroup regions is $\sim 2.4 \times 10^{-6} \AA^{-2}$, which decreased to $\sim 1.4 \times 10^{-6} \AA^{-2}$ upon addition of P3TMAHT. This may be attributed to the displacement of $\mathrm{D}_{2} \mathrm{O}$ molecules from the head-group region by the cationic poly(thiophene), which has a much lower SLD of $0.44 \times 10^{-6} \AA^{-2}$. The P3TMAHT/DPPC bilayers became slightly thinner and drier, but comparatively did not significantly change upon incorporation of the CPE. The P3Anionic/DPPC samples proved the most difficult to fit with the Core-Multi-Shell model. The excess scatter at $0.07 \AA^{-1}$ may be due to interparticle interactions between phospholipid vesicles or repulsion between similarly charged P3Anionic chains, however the fits were not found to improve significantly upon the addition of a structure factor. ${ }^{70}$ Nevertheless, fitting with this model gave vesicle bilayers with larger total thicknesses $(41.2 \AA)$ and larger head-group regions of $\sim 10.5 \AA$. The SLDs of P3Anionic and the DPPC head-groups are both $\sim 1.0 \times 10^{-6} \AA^{-2}$, therefore, when intimately mixed the two species are expected to appear as one thicker 'shell'. In addition, when P3Anionic and P3TMAHT are mixed with the DPPC vesicles, the calculated radii of the vesicle cores were found to increase only moderately by $\sim 7$ and $\sim 2 \AA$, respectively, in comparison to the P3Zwit/DPPC system which increased by $23 \AA$. For P3Zwit/DPPC samples the total bilayer thickness also decreased upon addition of P3Zwit decreasing to $32.8 \AA$, whilst the thickness of the head-group region remained relatively constant. The 'wetness' of the shells also remained 
effectively unchanged. These results suggest an increase in surface area of the vesicle bilayer upon addition of the P3Zwit, which may potentially be inserting throughout the DPPC bilayer, effectively spacing the phospholipids out, and thus increasing the overall surface area of the vesicle.

Table 3. Structural parameters obtained from the SANS data using the MKP method and fitting to the Core-Multi-Shell model. The error values for the MKP fits were calculated from the goodness-of-fit $\mathrm{R}^{2}$ values. $\phi_{\mathrm{dry}}$ is the calculated percentage volume of dry material that each CoreMulti-Shell model fit represents, an ideal fit for these samples has a $\phi_{\text {dry }}$ of $\sim 1$ vol\%.

\begin{tabular}{|c|c|c|c|c|c|}
\hline \multirow{2}{*}{\multicolumn{2}{|c|}{ Parameters }} & \multirow[b]{2}{*}{$\begin{array}{c}\text { Blank DPPC } \\
\text { vesicles }\end{array}$} & \multicolumn{3}{|c|}{ CPE:DPPC (1:1 charge ratio) } \\
\hline & & & P3Zwit & P3TMAHT & P3Anionic \\
\hline \multicolumn{2}{|c|}{ Bilayer thickness via MKP method $(\AA)$} & $35.3( \pm 0.5)$ & $33.3( \pm 0.1)$ & $34.5( \pm 0.1)$ & $43.3( \pm 0.1)$ \\
\hline \multicolumn{6}{|c|}{ Core-Multi-Shell model: } \\
\hline \multirow{2}{*}{$\begin{array}{l}\text { Inner } \\
\text { leaflet }\end{array}$} & Head-group thickness $(\AA)$ & 6.0 & 6.4 & 7.4 & 10.5 \\
\hline & Tail thickness $(\AA)$ & 13.0 & 10.0 & 10.5 & 10.1 \\
\hline \multirow{2}{*}{$\begin{array}{l}\text { Outer } \\
\text { leaflet }\end{array}$} & Tail thickness $(\AA)$ & 13.0 & 10.0 & 10.5 & 10.1 \\
\hline & Head-group thickness $(\AA)$ & 6.0 & 6.4 & 6.7 & 10.6 \\
\hline \multicolumn{2}{|c|}{ Total shell thickness $(\AA)$} & 38.0 & 32.8 & 35.1 & 41.2 \\
\hline \multicolumn{2}{|c|}{ Radius of the vesicle core $(\AA)$} & 481.3 & 504.0 & 483.0 & 489.9 \\
\hline \multirow{2}{*}{$\begin{array}{l}\text { Inner } \\
\text { leaflet }\end{array}$} & Head-group SLD $\left(\times 10^{-6} \AA^{-2}\right)$ & 2.44 & 2.43 & 1.38 & 0.96 \\
\hline & Tail SLD $\left(\times 10^{-6} \AA^{-2}\right)$ & 0.11 & 0 & 0 & -0.50 \\
\hline \multirow{2}{*}{$\begin{array}{l}\text { Outer } \\
\text { leaflet }\end{array}$} & Tail SLD $\left(\times 10^{-6} \AA^{-2}\right)$ & 0.11 & 0 & 0 & -0.50 \\
\hline & Head-group SLD $\left(\times 10^{-6} \AA^{-2}\right)$ & 2.35 & 2.27 & 1.38 & 0.96 \\
\hline \multicolumn{2}{|l|}{$\phi_{\mathrm{dy}}(\%)$} & 1.3 & 1.3 & 1.1 & 1.2 \\
\hline
\end{tabular}


Imaging Studies. Epi-fluorescence microscopy was performed on a mixture of CPE/DPPC multilamellar vesicles (MLVs) to gain further insight into the incorporation of the CPEs within the phospholipid bilayers. ${ }^{75}$ The P3Zwit/DPPC sample (Figure 6a) clearly shows red emission throughout the MLVs, suggesting the CPE is able to penetrate through multiple phospholipid bilayers. In contrast, the P3TMAHT/DPPC sample (Figure 6b) shows an uneven vesicle surface which may be attributed to aggregates of $\mathrm{CPE}$ on the vesicle, or potentially aggregation of the CPE/DPPC vesicles themselves. ${ }^{22}$ The vesicle structure is most dominant in the P3Anionic/DPPC samples (Figure 6c), with the poly(thiophene) emission localized on the outer layers of the MLVs.

Atomic force microscopy was used to measure the surface topography of the DPPC LUVs, CPEs and their mixtures..$^{76}$ AFM images of DPPC vesicles appeared as monodisperse spheres, $74( \pm 10) \mathrm{nm}$ in diameter (Figure S12a). The pure CPE samples gave large, amorphous aggregates which were $300-400 \mathrm{~nm}$ in diameter (Figure S12b-d). Each CPE/DPPC sample contained features of two population sizes. In all cases there was no evidence of the pure CPE aggregates. The smaller populations were spheres of $\sim 70 \mathrm{~nm}$ diameter, which potentially correspond to pure DPPC vesicles; however, it is more probable that these smaller features are DPPC vesicles which have only accommodated a few poly(thiophene) chains. The second, larger population in the P3TMAHT/DPPC sample (Figure 6e) featured spherical objects $\sim 187( \pm 27) \mathrm{nm}$ in diameter, with rough surfaces as indicated by SANS. A similar surface morphology has previously been reported for E.coli cells associated with cationic CPEs and was attributed to either a coating of $\mathrm{CPE}$ aggregates, disruption to the cell membrane, or a combination of both.22 Since the DLS studies performed here suggest that the vesicle structure is retained, we propose that the roughening of the DPPC vesicles is most likely due to the formation of P3TMAHT 
clusters on the vesicle surface or aggregates of the CPE-DPPC vesicles. In contrast, the larger features in the P3Zwit/DPPC films (Figure 6d) were smooth spheres with diameters of $177( \pm 41)$ $\mathrm{nm}$. The second population in P3Anionic/DPPC samples were aggregates $\sim 158( \pm 78) \mathrm{nm}$ in diameter, which clearly exhibit a multilayer effect (inset, Figure 6F). The outer shell has a thickness of $\sim 30 \mathrm{~nm}$, which could correspond to CPE chains extending from the vesicle surface, as suggested by the $\zeta$ measurements and the increase in head-group thickness observed using SANS. The large, smooth, spherical aggregates in the P3Zwit:DPPC and P3Anionic:DPPC samples are most likely the consequence of vesicle fusion. In particular, we note the appearance of fused vesicles in the P3Anionic/DPPC AFM and epi-fluorescence images (white arrows in Figure 6c/f). Examples of such fused vesicle may be harder to identify in the P3Zwit/DPPC samples since the poly(thiophene) penetrates throughout the MLV bilayers, making it difficult to distinguish one adjacent vesicle from another in the epi-fluorescence images. CPE-induced fusion of phospholipid vesicles has previously been observed between the anionic poly(phenylene ethylene), poly-[5-methoxy-2-(3-sulfopropoxy)-1,4-phenylene-vinylene] (MPSPPV), and the cationic phospholipid, dioleoyl-sn-glycero-3-phosphocholine (DOPC). ${ }^{25}$ It was postulated that partial penetration of the CPE chains within the DOPC bilayer induces strain, while CPE chains extending from the surface of the host vesicle are capable of bridging to an adjacent vesicle bilayer. When combined these two effects create the necessary proximity for fusion to occur. We note that while drying effects may also contribute to the vesicle fusion observed here, the larger hydrodynamic diameter determined for P3Anionic/DPPC (for which fusion is most prominent) by DLS on freshly prepared solutions ( $<10$ min old) may support the idea of vesicle association in solution at fairly short timescales. 

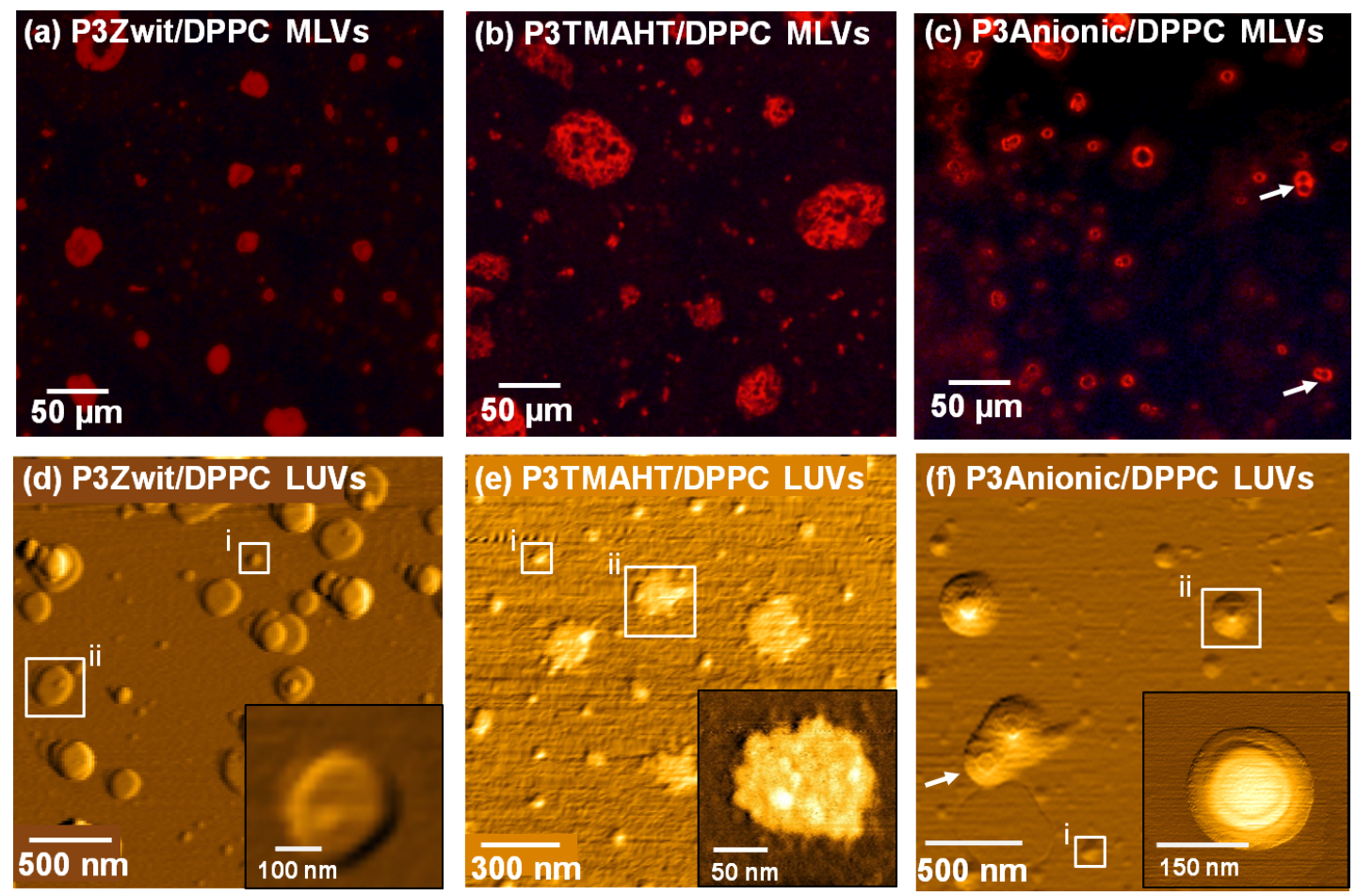

Figure 6. Epi-fluorescence images of DPPC multilamellar vesicles (MLVs) $\left(2.6 \times 10^{3} \mathrm{M}\right)$ titrated with (a) P3Zwit $\left(1.3 \times 10^{-3} \mathrm{M}\right.$ (r.u.)), (b) P3TMAHT $\left(1.3 \times 10^{3} \mathrm{M}\right.$ (r.u.)) and (c) P3Anionic $\left(1.3 \times 10^{3} \mathrm{M}\right.$ (r.u.)), $\lambda_{\mathrm{ec}}=435 \mathrm{~nm}$. AFM tapping mode images of (d) P3Zwit/DPPC, (e) P3TMAHT/DPPC and (f) P3Anionic/DPPC at 1:1 charge ratio $\left(2.0 \times 10^{s} \mathrm{M}\right)$ : (i) undoped DPPC vesicles, (ii) CPE-doped DPPC vesicle structures. Arrows highlight fused vesicles.

Mechanism of Interaction. From these studies three distinctive modes of interaction between each CPE and the DPPC vesicles are proposed, as shown by the schematic representation in Figure 7. The changes in the UV/Vis absorption and PL spectra confirm interaction between the CPEs and the vesicles. The calculated partition coefficients suggest the extent of incorporation follows the order P3Zwit > P3Anionic > P3TMAHT. In all cases an increase in size of the phospholipid vesicles was observed upon addition of the CPE (DLS, AFM, SANS); however, further inspection suggests that this is likely to be the result of different modes of interaction. 
Firstly, the global results suggest that P3Zwit, unrestricted by an overall charge, penetrates throughout the phospholipid bilayer. SANS revealed an increase in size of the vesicle including the core radius, suggesting an increase in the surface area of the phospholipid bilayer, which is to be expected if the CPE is incorporated throughout the thickness of the bilayer. In contrast, P3Anionic appears to only partially penetrate the phospholipid bilayer, burying within the phospholipid head-group region, encouraged by electrostatic attractive forces between the anionic sulfonate of the CPE and the outermost ammonium group of DPPC. This mechanism is supported by the increase in the phospholipid head-group thickness (SANS), efficient FRET with the membrane probe Prodan and localized changes to the outer surface of uni- and multilamellar vesicles (AFM, epi-fluorescence microscopy). Finally, the low $K_{p}$ value suggests that P3TMAHT has minimal penetration within the DPPC bilayer, presumably due to electrostatic repulsion between the positively-charged ammonium groups on both the CPE and DPPC, which results in the formation of extended CPE structures on the surface of the vesicles (PL enhancement, SANS, zeta potential, AFM, epi-fluorescence). All the measurements in this study were performed at room temperature and therefore the DPPC bilayers will exist in the lamellar gel phase..$^{32}$ However, we should also note that the steady-state emission of P3Anionic has previously been demonstrated to show remarkable sensitivity to membrane order. ${ }^{29}$ In contrast, both P3TMAHT and P3Zwit showed no sensitivity to the temperature-induced phase transitions of DPPC (see Figure S13). This is an encouraging result for the proposed assembly mechanisms, since P3Anionic is the only poly(thiophene) to specifically localize within the DPPC head-group region, where sensitivity to membrane order is believed to be the greatest." 


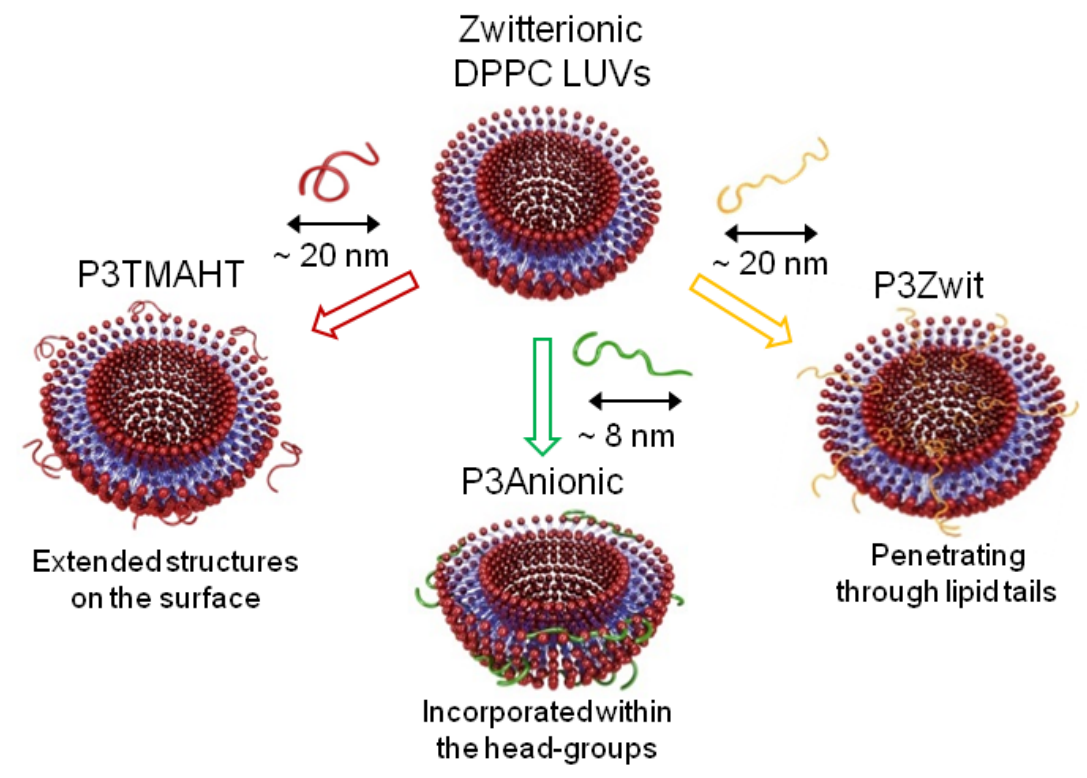

Figure 7. Schematic representation of the proposed CPE/DPPC vesicle interactions.

One potential reason for the observed difference in association between the charged poly(thiophene)s is their chain length. The anionic poly(thiophene) is approximately 21 repeat units (r.u.), in comparison the cationic poly(thiophene) is 49 r.u. Whitten et al. found that increasing the chain length of a cationic poly(phenylene ethynylene), decreased the degree of perturbation into the anionic phospholipid, DPPG, membrane ${ }^{26}$ However, it is important to note that P3Zwit has an identical chain-length to P3TMAHT, and yet is capable of burying deep within the bilayer, potentially suggesting that chain-length is not the mediating factor here. A further element to consider is the chemical structure of the DPPC phospholipid head-group, where the outermost group is a cationic trimethyl ammonium, as shown in Figure 1. Repulsive forces are expected to exist between this group and the cation of P3TMAHT, which may be preventing P3TMAHT from penetrating further within the zwitterionic head-group region. 


\section{CONCLUSIONS}

In summary, a strategic study of conjugated poly(thiophene)s bearing anionic, cationic and zwitterionic pendant groups has been performed to successfully demonstrate that the CPE penetration depth within a zwitterionic phospholipid bilayers can be controlled via chargemediation. With an overall neutral charge, the zwitterionic side-group of P3Zwit, in combination with its hydrophobic poly(thiophene) backbone, mean that P3Zwit can readily insert in between the DPPC zwitterionic head-groups, as well as uniquely the hydrophobic phospholipid tails. In contrast, both the charged poly(thiophene)s appear to localize around the head-group region of the phospholipid bilayers, with P3Anionic burying into the zwitterionic head-groups and P3TMAHT forming extended structures on the vesicle surface.

Previously, CPEs have been somewhat disregarded as viable fluorescent membrane probes due to their relatively large size and lack of accurate localization within the membrane bilayer. Yet we have shown that through the strategic selection of the CPE charge, the nature of their interaction with cell membranes can be tuned, without the need for complex molecular probe design, and the associated time-consuming experimental procedures. This has important consequences for a variety of applications, such as in biosensing, where the location of the probe is explicitly linked to biophysical properties under investigation ${ }^{15}$ and in drug delivery, where the presence of polymer shells has been shown to lead to prolonged drug delivery times..$^{7}$ The large polymer size is also known to advantageously inhibit cell internalization, ${ }^{79}$ which is a common problem for small molecule fluorescent dyes. ${ }^{80}$ At the same time, at only $<20 \mathrm{~nm}$ in length, the CPEs remain small enough to accurately probe the raft-like nanodomains in cell membranes (10$200 \mathrm{~nm}$ ), whose role and impact in cell function remains a topic of wide debate. ${ }^{81}$ Importantly, the P3Zwit-DPPC system also offers the unique advantage of a CPE-phospholipid system with the absence of mobile counterions. Such counterions are potentially problematic in biological 
sensing platforms due to their lack of biocompatibility or can result in undesirable side-reactions, such as fluorescence quenching by halide anions..$^{16}$ In conclusion, the selective engineering of CPE-phospholipid interfaces by strategic charge-mediation could play a key role in the design of superior biomedical and biotechnological devices.

\section{ASSOCIATED CONTENT}

Supporting Information. UV/Vis absorption and PL spectra of P3Anionic with DPPC vesicles, excitation and emission spectra of FRET studies with Prodan, $D_{\mathrm{n}}$ values determined using DLS, MKP fits of the SANS data, SANS data of pure CPEs, representative correlogram and phase diagram of DPPC, and AFM images of the pure CPEs and DPPC vesicles. This material is available free of charge via the Internet at http://pubs.acs.org.

\section{AUTHOR INFORMATION}

\section{Corresponding Author}

*E-mail: raevans@tcd.ie; Tel: +353 (0)1 8964215.

\section{Author Contributions}

The manuscript was written through contributions of all authors. All authors have given approval to the final version of the manuscript.

\section{Notes}

The authors declare no competing financial interest.

\section{ACKNOWLEDGMENT}


This work was supported by a Trinity Award postgraduate scholarship (J.E.H.). The authors would like to thank Dr Gavin McManus for assistance with epi-fluorescence microscopy. This research has been supported by the European Commission under the 7th Framework Programme through the 'Research Infrastructures' action of the 'Capacities' programme. Contract no: CPCSA_INFRA-2008- 1.1.1 Number 226507-NM13. We thank ISIS and STFC for the allocation of SANS Xpress beamtime.

\section{References}

1. Gennis, R. B., Biomembranes: Molecular Structure and Function. Springer-Verlag New York, Inc.: 1989, pp. 1.

2. Lingwood, D.; Simons, K. Lipid Rafts as a Membrane Organizing Principle. Science 2010, 327, 46-50.

3. $\quad$ Simons, K.; Ikonen, E. Functional Rafts in Cell Membranes. Nature 1997, 387, 569-572.

4. Jonsson, F.; Beke-Somfai, T.; Andréasson, J.; Nordén, B. Interactions of a Photochromic Spiropyran with Liposome Model Membranes. Langmuir 2013, 29, 2099-2103.

5. Needham, D.; Anyarambhatla, G.; Kong, G.; Dewhirst, M. W. A New TemperatureSensitive Liposome for Use with Mild Hyperthermia: Characterization and Testing in a Human Tumor Xenograft Model. Cancer Res. 2000, 60, 1197-1201.

6. Bolinger, P.-Y.; Stamou, D.; Vogel, H. Integrated Nanoreactor Systems: Triggering the Release and Mixing of Compounds inside Single Vesicles. J. Am. Chem. Soc. 2004, 126, 85948595.

7. Dent, M. R.; Lopez-Duarte, I.; Dickson, C. J.; Geoghegan, N. D.; Cooper, J. M.; Gould, I. R.; Krams, R.; Bull, J. A.; Brooks, N. J.; Kuimova, M. K. Imaging Phase Separation in Model Lipid Membranes through the Use of Bodipy Based Molecular Rotors. Phys. Chem. Chem. Phys. 2015, 17, 18393-18402.

8. Saxena, R.; Shrivastava, S.; Haldar, S.; Klymchenko, A. S.; Chattopadhyay, A. Location, Dynamics and Solvent Relaxation of a Nile Red-Based Phase-Sensitive Fluorescent Membrane Probe. Chem. Phys. Lipids 2014, 183, 1-8.

9. Muro, E.; Fragola, A.; Pons, T.; Lequeux, N.; Ioannou, A.; Skourides, P.; Dubertret, B. Comparing Intracellular Stability and Targeting of Sulfobetaine Quantum Dots with Other Surface Chemistries in Live Cells. Small 2012, 8, 1029-1037.

10. Sasaki, H.; White, S. H. A Novel Fluorescent Probe That Senses the Physical State of Lipid Bilayers. Biophys. J. 2009, 96, 4631-4641.

11. Prifti, E.; Reymond, L.; Umebayashi, M.; Hovius, R.; Riezman, H.; Johnsson, K. A Fluorogenic Probe for Snap-Tagged Plasma Membrane Proteins Based on the Solvatochromic Molecule Nile Red. ACS Chem. Biol. 2014, 9, 606-612.

12. Kilin, V.; Glushonkov, O.; Herdly, L.; Klymchenko, A.; Richert, L.; Mely, Y. Fluorescence Lifetime Imaging of Membrane Lipid Order with a Ratiometric Fluorescent Probe. Biophys. J. 2015, 108, 2521-2531. 
13. Bacalum, M.; Wang, L.; Boodts, S.; Yuan, P.; Leen, V.; Smisdom, N.; Fron, E.; Knippenberg, S.; Fabre, G.; Trouillas, P.; Beljonne, D.; Dehaen, W.; Boens, N.; Ameloot, M. A Blue-Light-Emitting Bodipy Probe for Lipid Membranes. Langmuir 2016, 32, 3495-3505.

14. Kucherak, O. A.; Oncul, S.; Darwich, Z.; Yushchenko, D. A.; Arntz, Y.; Didier, P.; Mély, Y.; Klymchenko, A. S. Switchable Nile Red-Based Probe for Cholesterol and Lipid Order at the Outer Leaflet of Biomembranes. J. Am. Chem. Soc. 2010, 132, 4907-4916.

15. Demchenko, A. P.; Mély, Y.; Duportail, G.; Klymchenko, A. S. Monitoring Biophysical Properties of Lipid Membranes by Environment-Sensitive Fluorescent Probes. Biophys. J. 2009, 96, 3461-3470.

16. Fonseca, S. M.; Galvão, R. P.; Burrows, H. D.; Gutacker, A.; Scherf, U.; Bazan, G. C. Selective Fluorescence Quenching in Cationic Fluorene-Thiophene Diblock Copolymers for Ratiometric Sensing of Anions. Macromol. Rapid Commun. 2013, 34, 717-722.

17. Chemburu, S.; Ji, E.; Casana, Y.; Wu, Y.; Buranda, T.; Schanze, K. S.; Lopez, G. P.; Whitten, D. G. Conjugated Polyelectrolyte Supported Bead Based Assays for Phospholipase A2 Activity. J. Phys. Chem. B 2008, 112, 14492-14499.

18. Kahveci, Z.; Martínez-Tomé, M. J.; Mallavia, R.; Mateo, C. R. Use of the Conjugated Polyelectrolyte Poly $\{[9,9$-bis(6'- $N, N, N$-trimethylammonium)hexyl]fluorene-phenylene $\}$. Bromide (HTMA-PFP) as a Fluorescent Membrane Marker. Biomacromolecules 2013, 14, 19901998.

19. Evans, R. C.; Knaapila, M.; Willis-Fox, N.; Kraft, M.; Terry, A.; Burrows, H. D.; Scherf, U. Cationic Polythiophene-Surfactant Self-Assembly Complexes: Phase Transitions, Optical Response, and Sensing. Langmuir 2012, 28, 12348-12356.

20. Evans, R. C. Harnessing Self-Assembly Strategies for the Rational Design of Conjugated Polymer Based Materials. J. Mater. Chem. C 2013, 1, 4190-4200.

21. Anwar, N.; Rix, A.; Lederle, W.; Kuehne, A. J. C. Rgd-Decorated Conjugated Polymer Particles as Fluorescent Biomedical Probes Prepared by Sonogashira Dispersion Polymerization. Chem. Commun. 2015, 51, 9358-9361.

22. Edens, L. E.; Wang, Y.; Whitten, D. G.; Keller, D. J. AFM Images of the Dark Biocidal Action of Cationic Conjugated Polyelectrolytes and Oligomers on Escherichia Coli. Langmuir 2014, 30, 10691-10697.

23. Du, J.; Thomas, A. W.; Chen, X.; Garner, L. E.; Vandenberg, C. A.; Bazan, G. C. Increased Ion Conductance across Mammalian Membranes Modified with Conjugated Oligoelectrolytes. Chem. Commun. 2013, 49, 9624-9626.

24. $\quad$ Ding, L.; Chi, E. Y.; Chemburu, S.; Ji, E.; Schanze, K. S.; Lopez, G. P.; Whitten, D. G. Insight into the Mechanism of Antimicrobial Poly(Phenylene Ethynylene) Polyelectrolytes: Interactions with Phosphatidylglycerol Lipid Membranes. Langmuir 2009, 25, 13742-13751.

25. Karam, P.; Hariri, A. A.; Calver, C. F.; Zhao, X.; Schanze, K. S.; Cosa, G. Interaction of Anionic Phenylene Ethynylene Polymers with Lipids: From Membrane Embedding to Liposome Fusion. Langmuir 2014, 30, 10704-10711.

26. Wang, Y.; Jones, E. M.; Tang, Y.; Ji, E.; Lopez, G. P.; Chi, E. Y.; Schanze, K. S.; Whitten, D. G. Effect of Polymer Chain Length on Membrane Perturbation Activity of Cationic Phenylene Ethynylene Oligomers and Polymers. Langmuir 2011, 27, 10770-10775.

27. Vermette, P.; Meagher, L. Interactions of Phospholipid- and Poly(Ethylene Glycol)Modified Surfaces with Biological Systems: Relation to Physico-Chemical Properties and Mechanisms. Colloid. Surf. B 2003, 28, 153-198. 
28. Costa, T.; de Azevedo, D.; Stewart, B.; Knaapila, M.; Valente, A. J. M.; Kraft, M.; Scherf, U.; Burrows, H. D. Interactions of a Zwitterionic Thiophene-Based Conjugated Polymer with Surfactants. Polym. Chem. 2015, 6, 8036-8046.

29. Houston, J. E.; Kraft, M.; Scherf, U.; Evans, R. C. Sequential Detection of Multiple Phase Transitions in Model Biological Membranes Using a Red-Emitting Conjugated Polyelectrolyte. Phys. Chem. Chem. Phys. 2016, 18, 12423-12427.

30. Zhai, L.; McCullough, R. D. Layer-by-Layer Assembly of Polythiophene. Adv. Mater. 2002, 14, 901-905.

31. Kraft, M.; Adamczyk, S.; Polywka, A.; Zilberberg, K.; Weijtens, C.; Meyer, J.; Görrn, P.; Riedl, T.; Scherf, U. Polyanionic, Alkylthiosulfate-Based Thiol Precursors for Conjugated Polymer Self-Assembly onto Gold and Silver. ACS Appl. Mater. Interfaces 2014, 6, 1175811765.

32. Matsuki, H.; Goto, M.; Tada, K.; Tamai, N. Thermotropic and Barotropic Phase Behavior of Phosphatidylcholine Bilayers. Inter. J. Mol. Sci. 2013, 14, 2282-2302.

33. International Standard Iso22412 Particle Size Analysis - Dynamic Light Scattering. International Organisation for Standardisation (ISO): 2008.

34. Hunter, J. R., Zeta Potential in Colloid Science. Academic Press: London, 1981

35. Pucci, C.; Scipioni, A.; La Mesa, C. Albumin Binding onto Synthetic Vesicles. Soft Matter 2012, 8, 9669-9675.

36. http://www.mantidproject.org.

37. Wignall, G. D.; Bates, F. S. Absolute Calibration of Small-Angle Neutron Scattering Data. J. Appl. Crystallogr. 1987, 20, 28-40.

38. Schmidt, P. W., Some Fundamental Concepts and Techniques Useful in Small-Angle Scattering Studies of Disordered Solids. In Modern Aspects of Small-Angle Scattering. Kluwer Academic Publishers: Dordrecht: The Netherlands, 1995.

39. Kiselev, M. A.; Zemlyanaya, E. V.; Aswal, V. K.; Neubert, R. H. H. What Can We Learn About the Lipid Vesicle Structure from the Small-Angle Neutron Scattering Experiment? Eur. Biophys. J. 2006, 35, 477-493.

40. Balgavý, P.; Dubničková, M.; Kučerka, N.; Kiselev, M. A.; Yaradaikin, S. P.; Uhrı’ková, D. Bilayer Thickness and Lipid Interface Area in Unilamellar Extruded 1,2Diacylphosphatidylcholine Liposomes: A Small-Angle Neutron Scattering Study. Biochim. Biophys. Acta 2001, 1512, 40-52.

41. http://www.sasview.org/index.html.

42. Qian, S.; Heller, W. T. Peptide-Induced Asymmetric Distribution of Charged Lipids in a Vesicle Bilayer Revealed by Small-Angle Neutron Scattering. J. Phys. Chem. B 2011, 115, 9831-9837.

43. Coutinho, A.; Prieto, M. Self-Association of the Polyene Antibiotic Nystatin in Dipalmitoylphosphatidylcholine Vesicles: A Time-Resolved Fluorescence Study. Biophys. J. 1995, 69, 2541-2557.

44. Santos, N. C.; Prieto, M.; Castanho, M. A. R. B. Quantifying Molecular Partition into Model Systems of Biomembranes: An Emphasis on Optical Spectroscopic Methods. Biochim. Biophys. Acta 2003, 1612, 123-135.

45. Vermeir, M.; Boens, N.; Heirwegh, K. P. Interaction of 7-N-Alkoxycoumarins with Cytochrome P-450(2) and Their Partitioning into Liposomal Membranes. Assessment of Methods for Determination of Membrane Partition Coefficients. Biochem. J. 1992, 284, 483-490. 
46. Greenwood, A. I.; Tristram-Nagle, S.; Nagle, J. F. Partial Molecular Volumes of Lipids and Cholesterol. Chem. Phys. Lipids 2006, 143, 1-10.

47. Lakowics, J. R., Principles of Fluorescence Spectorscopy. Springer: 2006, pp. 443-472.

48. Kozyra, K. A.; Heldt, J. R.; Engelke, M.; Diehl, H. A. Phase Transition Affects Energy Transfer Efficiency in Phospholipid Vesicles. Spectrochim. Acta Part A 2005, 61, 1153-1161.

49. Swager, T. M.; Gil, C. J.; Wrighton, M. S. Fluorescence Studies of Poly(PPhenyleneethynylene)S: The Effect of Anthracene Substitution. J. Phys. Chem. 1995, 99, 48864893.

50. Kahveci, Z.; Martínez-Tomé, M.; Esquembre, R.; Mallavia, R.; Mateo, C. Selective Interaction of a Cationic Polyfluorene with Model Lipid Membranes: Anionic Versus Zwitterionic Lipids. Materials 2014, 7, 2120-2140.

51. Costa, T.; Garner, L. E.; Knaapila, M.; Thomas, A. W.; Rogers, S. E.; Bazan, G. C.; Burrows, H. D. Aggregation Properties of P-Phenylene Vinylene Based Conjugated Oligoelectrolytes with Surfactants. Langmuir 2013, 29, 10047-10058.

52. $\quad$ Burrows, H. D.; Tapia, M. a. J.; Fonseca, S. M.; Pradhan, S.; Scherf, U.; Silva, C. u. L.; Pais, A. A. C. C.; Valente, A. J. M.; Schillén, K.; Alfredsson, V.; Carnerup, A. M.; Tomšič, M.; Jamnik, A. Solubilization of Poly\{1,4-Phenylene-[9,9-Bis(4-Phenoxy-Butylsulfonate)]Fluorene2,7-Diyl\} in Water by Nonionic Amphiphiles. Langmuir 2009, 25, 5545-5556.

53. Stokes, K. K.; Heuzé, K.; McCullough, R. D. New Phosphonic Acid Functionalized, Regioregular Polythiophenes. Macromolecules 2003, 36, 7114-7118.

54. Tan, C.; Atas, E.; Müller, J. G.; Pinto, M. R.; Kleiman, V. D.; Schanze, K. S. Amplified Quenching of a Conjugated Polyelectrolyte by Cyanine Dyes. J. Am. Chem. Soc. 2004, 126, 13685-13694.

55. Ghatak, C.; Rao, V. G.; Pramanik, R.; Sarkar, S.; Sarkar, N. The Effect of Membrane Fluidity on FRET Parameters: An Energy Transfer Study inside Small Unilamellar Vesicle. Phys. Chem. Chem. Phys. 2011, 13, 3711-3720.

56. Parasassi, T.; Krasnowska, E.; Bagatolli, L.; Gratton, E. Laurdan and Prodan as PolaritySensitive Fluorescent Membrane Probes. J. Fluoresc. 1998, 8, 365-373.

57. Förster, T. Zwischenmolekulare Energiewanderung und Fluoreszenz. Annalen der Physik 1948, 437, 55-75.

58. Beljonne, D.; Pourtois, G.; Silva, C.; Hennebicq, E.; Herz, L. M.; Friend, R. H.; Scholes, G. D.; Setayesh, S.; Müllen, K.; Brédas, J. L. Interchain vs. Intrachain Energy Transfer in Acceptor-Capped Conjugated Polymers. Proc. Nat. Acad. Sci. 2002, 99, 10982-10987.

59. Wong, K. F.; Bagchi, B.; Rossky, P. J. Distance and Orientation Dependence of Excitation Transfer Rates in Conjugated Systems: Beyond the Förster Theory. J. Phys. Chem. A 2004, 108, 5752-5763.

60. Jiang, H.; Taranekar, P.; Reynolds, J. R.; Schanze, K. S. Conjugated Polyelectrolytes: Synthesis, Photophysics, and Applications. Angew. Chem. Int. Ed. 2009, 48, 4300-4316.

61. A representation correlogram and phase diagram for the DLS and zeta potential measurements, respectively, are available in the Supporting Information.

62. Ngo, A. T.; Cosa, G. Assembly of Zwitterionic Phospholipid/Conjugated Polyelectrolyte Complexes: Structure and Photophysical Properties. Langmuir 2009, 26, 6746-6754.

63. Sybachin, A. V.; Efimova, A. A.; Litmanovich, E. A.; Menger, F. M.; Yaroslavov, A. A. Complexation of Polycations to Anionic Liposomes: Composition and Structure of the Interfacial Complexes. Langmuir 2007, 23, 10034-10039. 
64. Wang, Y.; Tang, Y.; Zhou, Z.; Ji, E.; Lopez, G. P.; Chi, E. Y.; Schanze, K. S.; Whitten, D. G. Membrane Perturbation Activity of Cationic Phenylene Ethynylene Oligomers and Polymers: Selectivity against Model Bacterial and Mammalian Membranes. Langmuir 2010, 26, 12509-12514.

65. Fan, H. Y.; Nazari, M.; Raval, G.; Khan, Z.; Patel, H.; Heerklotz, H. Utilizing Zeta Potential Measurements to Study the Effective Charge, Membrane Partitioning, and Membrane Permeation of the Lipopeptide Surfactin. Biochim. Biophys. Acta 2014, 1838, 2306-2312.

66. Wiącek, A. E. Investigations of DPPC Effect on A12O3 Particles in the Presence of (Phospho)Lipases by the Zeta Potential and Effective Diameter Measurements. Appl. Surf. Sci. 2011, 257, 4495-4504.

67. Kepczynski, M.; Jamróz, D.; Wytrwal, M.; Bednar, J.; Rzad, E.; Nowakowska, M. Interactions of a Hydrophobically Modified Polycation with Zwitterionic Lipid Membranes. Langmuir 2011, 28, 676-688.

68. Grillo, I.; Kats, E. I.; Muratov, A. R. Formation and Growth of Anionic Vesicles Followed by Small-Angle Neutron Scattering. Langmuir 2003, 19, 4573-4581.

69. Thomas, A.; Houston, J. E.; Van den Brande, N.; De Winter, J.; Chevrier, M.; Heenan, R. K.; Terry, A. E.; Richeter, S.; Mehdi, A.; Van Mele, B.; Dubois, P.; Lazzaroni, R.; Gerbaux, P.; Evans, R. C.; Clément, S. All-Conjugated Cationic Copolythiophene "Rod-Rod" Block Copolyelectrolytes: Synthesis, Optical Properties and Solvent-Dependent Assembly. Polym. Chem. 2014, 5, 3352-3362.

70. $\quad$ Knaapila, M.; Evans, R. C.; Gutacker, A.; Garamus, V. M.; Szekely, N. K.; Scherf, U.; Burrows, H. D. Conjugated Polyelectrolyte (CPE) Poly[3-[6-( $N$-Methylimidazolium)hexyl]-2,5thiophene] Complexed with Aqueous Sodium Dodecylsulfate Amphiphile: Synthesis, Solution Structure and "Surfactochromic" Properties. Soft Matter 2011, 7, 6863-6872.

71. Hammouda, B. A New Guinier-Porod Model. J. Appl. Crystallogr. 2010, 43, 716-719.

72. Pencer, J.; Hallett, F. R. Small-Angle Neutron Scattering from Large Unilamellar Vesicles: An Improved Method for Membrane Thickness Determination. Phys. Rev. E 2000, 61, 3003-3008.

73. Satoh, K. Determination of Binding Constants of $\mathrm{Ca}^{2+}, \mathrm{Na}^{+}$, and $\mathrm{Cl}^{-}$Ions to Liposomal Membranes of Dipalmitoylphosphatidylcholine at Gel Phase by Particle Electrophoresis. Biochim. Biophys. Acta 1995, 1239, 239-248.

74. Uhríková, D.; Kučerka, N.; Teixeira, J.; Gordeliy, V.; Balgavý, P. Structural Changes in Dipalmitoylphosphatidylcholine Bilayer Promoted by $\mathrm{Ca}^{2+}$ Ions: A Small-Angle Neutron Scattering Study. Chem. Phys. Lipids 2008, 155, 80-89.

75. Larger MLVs (5-20 $\mu \mathrm{m}$ in diameter) were used for epi-fluorescence microscopy measurements due to the limited size resolution of this technique. The preparation procedure for MLVs is described in the Supporting Information.

76. The AFM samples were approx. five times more dilute than the SANS. So we would expect even fewer vesicle-vesicle interactions in our AFM images.

77. Shrivastava, S.; Haldar, S.; Gimpl, G.; Chattopadhyay, A. Orientation and Dynamics of a Novel Fluorescent Cholesterol Analogue in Membranes of Varying Phase. J. Phys. Chem. B 2009, 113, 4475-4481.

78. Mohr, K.; Müller, S. S.; Müller, L. K.; Rusitzka, K.; Gietzen, S.; Frey, H.; Schmidt, M. Evaluation of Multifunctional Liposomes in Human Blood Serum by Light Scattering. Langmuir 2014, 30, 14954-14962. 
79. Tapia, M. J.; Monteserin, M.; Burrows, H. D.; Almeida, J. A. S.; Pais, A. A. C. C.; Pina, J.; Seixas de Melo, J. S.; Jarmelo, S.; Estelrich, J. From Molecular Modelling to Photophysics of Neutral Oligo- and Polyfluorenes Incorporated into Phospholipid Bilayers. Soft Matter 2015, 11, 303-317.

80. Wang, H.-Y.; Jia, H.-R.; Lu, X.; Chen, B.; Zhou, G.; He, N.; Chen, Z.; Wu, F.-G. Imaging Plasma Membranes without Cellular Internalization: Multisite Membrane Anchoring Reagents Based on Glycol Chitosan Derivatives. J. Mater. Chem. B 2015, 3, 6165-6173

81. Marin, R.; Rojo, J. A.; Fabelo, N.; Fernandez, C. E.; Diaz, M. Lipid Raft Disarrangement as a Result of Neuropathological Progresses: A Novel Strategy for Early Diagnosis? Neuroscience 2013, 245, 26-39. 


\section{Table of Contents}

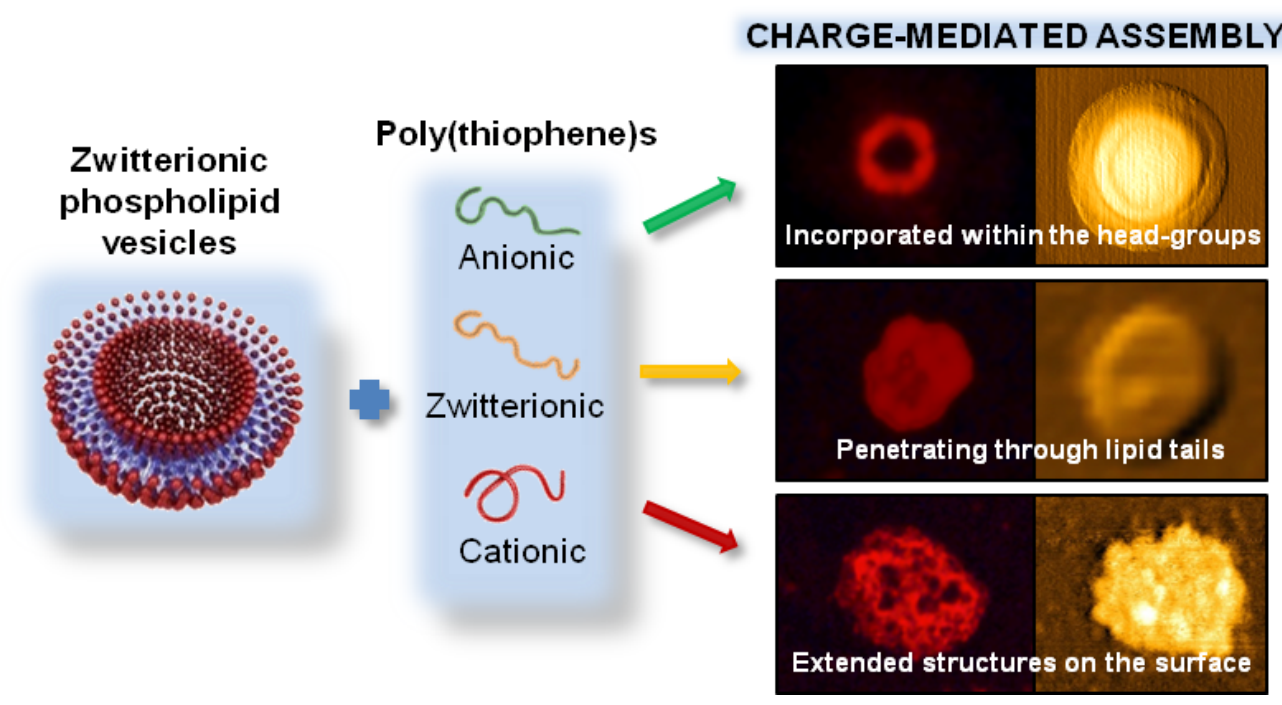

A strategic study of conjugated poly(thiophene)s bearing anionic, cationic and zwitterionic pendant groups successfully demonstrates that controlled polymer penetration within zwitterionic phospholipid bilayers can be achieved via charge-mediation. 